\title{
Bioelectrical Interfaces with Cortical Spheroids in Three-Dimensions
}

Anna Kalmykov ${ }^{1, \S}$, Jay W. Reddy ${ }^{2, \S}$, Esther Bedoyan ${ }^{2, \S}$, Yingqiao Wang ${ }^{3}$, Raghav Garg ${ }^{3}$, Sahil K. Rastogi ${ }^{1}$, Devora Cohen-Karni ${ }^{4}$, Maysamreza Chamanzar ${ }^{2, *}$, Tzahi Cohen-Karni ${ }^{1,3, *}$

${ }^{1}$ Department of Biomedical Engineering, Carnegie Mellon University, Pittsburgh, PA- 15213, USA.

${ }^{2}$ Department of Electrical and Computer Engineering, Carnegie Mellon University, Pittsburgh, PA- 15213, USA.

${ }^{3}$ Department of Materials Science and Engineering, Carnegie Mellon University, Pittsburgh, PA15213, USA.

${ }^{4}$ Preclinical education, Lake Erie College of Osteopathic Medicine at Seton Hill, Greensburg, PA15601, USA.

$\S$ These authors contributed equally to this work.

* Corresponding authors: Tzahi Cohen-Karni tzahi@andrew.cmu.edu; Maysamreza Chamanzar mchamanz@andrew.cmu.edu 


\begin{abstract}
Three-dimensional (3D) neuronal spheroid culture serves as a powerful model system for the investigation of neurological disorders and drug discovery. The success of such a model system requires techniques that enable high-resolution functional readout across the entire spheroid. Conventional microelectrode arrays and implantable neural probes cannot monitor the electrophysiology activity across the entire native 3D geometry of the cellular construct. Here, we demonstrate a 3D self-rolled biosensor array (3D-SR-BA) integrated with a 3D cortical spheroid culture for simultaneous in-vitro electrophysiology recording, functional $\mathrm{Ca}^{2+}$ imaging, and drug effect monitoring. We have also developed a signal processing pipeline to detect neural firings with high spatiotemporal resolution from the electrophysiology recordings based on established spike sorting methods. The 3D-SR-BAs cortical spheroid interface provides a stable, high sensitivity recording of neural action potentials $(<50 \mu \mathrm{V}$ peak-to-peak amplitude). The 3D-SRBA is demonstrated as a potential drug screening platform through the investigation of the neural response to the excitatory neurotransmitter glutamate. Upon addition of glutamate, the neuronal firing rates increased notably corresponding well with the functional $\mathrm{Ca}^{2+}$ imaging. Our entire system, including the 3D-SR-BA integrated with neural spheroid culture, enables simultaneous electrophysiology recording and functional $\mathrm{Ca}^{2+}$ imaging with high spatiotemporal resolution in conjunction with chemical stimulation. We demonstrate a powerful toolset for future studies of tissue development, disease progression, and drug testing and screening, especially when combined with native spheroid cultures directly extracted from humans.
\end{abstract}




\section{Introduction}

Three dimensional (3D) cell culture closely mimics the physiology and development of native tissue and serves as a powerful system for disease modeling and designing new therapeutics. ${ }^{1-7}$ Spheroids, the most common 3D tissue model, are based on the cells' tendency to aggregate, forgoing a scaffold or extracellular matrix and thus reducing the batch-to-batch variation. ${ }^{8}$ 3D cultures of neuronally differentiated stem cells have enabled in vitro modeling of diseases such as autism, ${ }^{3}$ microcephaly, ${ }^{6}$ and Alzheimer's disease. ${ }^{4,9}$ The success of these model systems requires techniques to probe the rich structure and function of the $3 \mathrm{D}$ cellular construct with high-resolution functional readout.

$3 \mathrm{D}$ cell culture holds a great promise in personalized medicine, ${ }^{1-6}$ as it bridges the gap between 2D cell culture and in vivo testing. For example, 2D in vitro models of the central nervous system (CNS), do not recapitulate axonal regeneration, while in 3D models, synaptic connections and myelinated axons are formed. ${ }^{10}$ Therefore, proper neurodegenerative disease modeling necessitates $3 \mathrm{D}$ tissue models. ${ }^{2,4-5,10}$ Using human derived in vivo-like $3 \mathrm{D}$ tissue models may reduce animal testing in drug toxicology studies. ${ }^{8}$ For neural constructs, functionality is most accurately characterized by recording neuronal electrophysiology (ephys). ${ }^{10}$ Recording the electrical activity of neural spheroids in their native 3D architecture over time with high spatial and temporal resolution is critical for developing disease models and validating potential treatments. ${ }^{10}$

3D neural structures have been investigated by conventional neural recording techniques. Patch clamp is limited to recordings from sectioned organoids, and from a few individual cells. ${ }^{9}$ High-density implantable neural probes have been used to record neural activity in the probe vicinity. ${ }^{11}$ Two-dimensional (2D) microelectrode arrays (MEAs) have been used to interface with 
neuronal organoids but exhibit a limited $2 \mathrm{D}$ interface with the organoid. ${ }^{12-13}$ Optical techniques, such as functional $\mathrm{Ca}^{2+}$ imaging, which provide high spatial resolution and throughput, lack the temporal precision of ephys techniques (msec resolution), required to study the temporal dynamics of neural activity. ${ }^{14}$

Here, we present a platform consisting of a 3D self-rolled biosensor array (3D-SR-BA) ${ }^{15}$ that wraps around the $3 \mathrm{D}$ structure of a rat cortical spheroid. We demonstrate a platform for studying 3D neural spheroids, including cell culture, 3D self-rolling electrode arrays (Figure 1 A I, II), chemical neuronal stimulation, simultaneous ephys recording, and functional $\mathrm{Ca}^{2+}$ imaging. We have developed a complete signal processing pipeline to analyze ephys recordings in conjunction with functional $\mathrm{Ca}^{2+}$ imaging of the neural activity across different regions of the spheroid in response to glutamate stimulation. The pipeline includes identification of localized neural activity and isolation of single units using spike sorting analysis to obtain firing rates of individual neurons (Figure 1 A III, IV). The demonstrated results in this paper exemplify the utility of this powerful platform for studying the effect of chemical stimulation (i.e., glutamate) on the neuronal response in spheroids with high spatiotemporal resolution using distributed ephys recording and high throughput $\mathrm{Ca}^{2+}$ imaging. Ephys recording using 3D-SR-BA will allow unprecedented access to the $3 \mathrm{D}$ structure of the organoid for recording neuronal activity with high temporal resolution, while functional $\mathrm{Ca}^{2+}$ imaging provides a complementary readout mechanism to capture the holistic activity of the tissue with high spatial resolution within the field of view. This novel platform provides tissue accessibility and high throughput long-term 3D neuronal recording, enabling exciting future biological applications, such as drug screening for neurological disorders. 


\section{Materials and Methods}

\section{D sensor array fabrication}

The 3D-SR-BAs were fabricated following a previously published microfabrication process, outlined in Figure S1. ${ }^{15-17} \mathrm{Si}$ substrates with a $600 \mathrm{~nm}$ wet thermal oxide (p-type, $\leq 0.005 \Omega \mathrm{cm}$, Nova Electronic Materials Ltd., catalog no. CP02 11208-OX) were cleaned with acetone and isopropyl alcohol (IPA) in an ultrasonic bath for 5 min each, $\mathrm{N}_{2}$ blow dried, and treated with $\mathrm{O}_{2}$ plasma at $100 \mathrm{~W}$ power for $1 \mathrm{~min}$ (Barrel Asher, International Plasma Corporation). The substrate was coated with $300 \mathrm{~nm}$ lift-off resist (LOR3A, Kayaku Advanced Materials) and $500 \mathrm{~nm}$ positive photoresist (Shipley S1805, Kayaku Advanced Materials). Outer electrode interconnects were defined by ultraviolet (UV) lithography using a mask aligner (MA6, SUSS MicroTec SE) followed by development for $1 \mathrm{~min}$ (CD26 developer, Kayaku Advanced Materials). $10 \mathrm{~nm}$ Cr (99.99\%, R.D. Mathis Co.) and $75 \mathrm{~nm} \mathrm{Au} \mathrm{(99.999 \% ,} \mathrm{Praxair)} \mathrm{were} \mathrm{deposited} \mathrm{using} \mathrm{thermal} \mathrm{evaporation}$ (Covap II, Angstrom Engineering Inc) as the interconnect metals. The LOR3A/Shipley 1805/metal stack was lifted off using Remover PG (Kayaku Advanced Materials) to pattern the electrode interconnects. The wafer was washed with acetone and IPA, $\mathrm{N}_{2}$ blow-dried, and treated with $\mathrm{O}_{2}$ plasma at $50 \mathrm{~W}$ power for $1 \mathrm{~min}$. A $200 \mathrm{~nm}$ Ge (99.999\%, Kurt J. Lesker Company) sacrificial layer was patterned and deposited using the same lift-off process. The mechanical support for the biosensors was patterned using negative photoresist (SU-8 2000.5, Kayaku Advanced Materials). Using a thinner (SU-8 2000 thinner, Kayaku Advanced Materials), the SU-8 composition was adjusted to $9.5 \%$ solids to achieve $200 \mathrm{~nm} \mathrm{SU-8} \mathrm{thickness.} \mathrm{The} \mathrm{biosensor} \mathrm{array} \mathrm{interconnects} \mathrm{were}$ patterned using similar methods as the outer interconnects. 1nm Cr (99.998\%, Kurt J. Lesker Company) / 50nm Pd (99.99\%, Kurt J. Lesker Company) / 25 nm Cr (99.998\%, Kurt J Lesker) / $10 \mathrm{~nm} \mathrm{Au} \mathrm{(99.999 \% ,} \mathrm{Kurt} \mathrm{J} \mathrm{Lesker)} \mathrm{were} \mathrm{deposited} \mathrm{using} \mathrm{an} \mathrm{electron} \mathrm{beam} \mathrm{evaporator} \mathrm{(Pro} \mathrm{Line}$ 
PVD Evaporator, Kurt J. Lesker Company). Lastly, the interconnects of the biosensors were passivated with $100 \mathrm{~nm}$ of SU-8 2000.5 diluted with SU-8 thinner to 7.15\% solids, patterned using photolithography to open the electrode sites (Figure S1).

\section{D-SR-BA structure release}

Post fabrication, the 3D-SR-BA chips were cleaned with acetone, IPA, and $\mathrm{N}_{2}$ blow dried. A rectangular chamber was prepared by using 10:1 base:curing agent polydimethylsiloxane (PDMS) (Sylgard 184 Silicone Elastomer, Dow Corning), cured overnight at $65^{\circ} \mathrm{C}$, cut to the needed dimensions and positioned on a device chip, such that the well surrounds the 3D-SR-BA region. A $1 \% \mathrm{H}_{2} \mathrm{O}_{2}$ (catalog no. 216763, Sigma-Aldrich) solution in deionized (DI) water was added to the well for 1 hour up-to overnight to dissolve the sacrificial layer and trigger self-rolling. After 3D-SR-BAs conformed in 3D, the solution was exchanged for DI water. The radius of curvature was estimated from a slice of 3D confocal microscopy image stack as depicted in Figure S1. Poly(3,4-ethylenedioxythiophene) : poly(sodium 4-styrenesulfonate) electrodeposition

3D-SR-BA modification with poly(3,4-ethylenedioxythiophene) : poly(sodium 4styrenesulfonate) (PEDOT:PSS) reduces the MEA's impedance, which results in higher signal amplitudes of ephys recordings. ${ }^{18}$ Electrodeposition was performed in a three-electrode cell setup using a potentiostat (Gamry R600+, Gamry Instruments). A Pt wire, Ag/AgCl electrode and Au microelectrode were used as counter, reference, and working electrodes, respectively. A solution of $0.01 \mathrm{M}$ ethylenedioxythiophene $(\mathrm{EDOT})^{18}(97 \%$, catalog no. 483028, Sigma-Aldrich) and 0.02 M poly(sodium 4-styrenesulfonate) (PSS) (catalog no. 243051, Sigma-Aldrich) was prepared in DI water. PEDOT:PSS electrodeposition was performed by using a constant current density of 0.5 $\mathrm{mA} / \mathrm{cm}^{2}$ applied for $10 \mathrm{~min}$ to each electrode sequentially. 


\section{Electrochemical characterization of microelectrodes}

To characterize the impedance of the electrodes before and after PEDOT:PSS electrodeposition, electrochemical impedance spectroscopy (EIS) was performed in a threeelectrode electrochemical cell using a potentiostat (Gamry R600+, Gamry Instruments). ${ }^{17} 1 \mathrm{X}$ Phosphate Buffered Saline (PBS) (catalog no. 10010023, ThermoFisher) was used as an electrolyte solution. A Pt wire, an $\mathrm{Ag} / \mathrm{AgCl}$ electrode and an $\mathrm{Au}$ microelectrode were used as counter, reference, and working electrodes, respectively. The frequency was scanned from $10 \mathrm{~Hz}$ to 100,00 Hz with $\mathrm{V}_{\mathrm{DC}}$ of $0 \mathrm{~V}$ and $\mathrm{V}_{\mathrm{AC}}$ of $10 \mathrm{mV}$. EIS experiments $(\mathrm{n}=11)$ were performed before and after PEDOT:PSS electrodeposition. Measurements were performed in a Faraday cage.

\section{Cortical neurons isolation}

Cortical neurons were obtained from primary embryonic day 18 (E18) rat cortical tissue from BrainBits LLC (SKU: SDECX). For cortical tissue dissociate, $2 \mathrm{~mL}$ media from the tissue's vial was aliquoted and stored for a later step. The tissue was incubated with a solution of $0.25 \%$ trypsin ethylenediaminetetraacetic acid (EDTA) (catalog no. 25200056, ThermoFisher) for 5 min. The tissue was again incubated with a solution of $0.25 \%$ trypsin EDTA supplemented with $0.1 \%$ Deoxyribonuclease (DNase, catalog no. EN0521, ThermoFisher) for another 5 min. Post incubation, the solution was replaced by the previously removed media. The tissue was physically dissociated by triturating it 5 times using a sterile fire-polished glass Pasteur pipette ca. $1 \mathrm{~mm}$ in diameter. This was followed by another set of trituration ( 5 times) with a narrower pipette (ca. 1/2$3 / 4 \mathrm{~mm}$ in diameter). The dissociated cells were then centrifuged at $1100 \mathrm{rpm}$ for $1 \mathrm{~min}$ and resuspended in $1 \mathrm{~mL}$ of fresh Neurobasal media (catalog no. A1413701, ThermoFisher) supplemented with $1 \times$ B27 (catalog no. A1413701, ThermoFisher), 1\% Glutamax (catalog no. 
35050-061, ThermoFisher) and 1\% Penicillin/Streptomycin (catalog no. 15140122, ThermoFisher).

\section{Cortical spheroid formation}

Cortical spheroids were formed following previously established protocols. ${ }^{19-20}$ Briefly, sterile $10 \mathrm{~g} / \mathrm{L}$ agarose solution was prepared by mixing agarose powder (catalog no. 16500, Invitrogen) with a solution of $0.9 \%(\mathrm{w} / \mathrm{v}) \mathrm{NaCl}$ (catalog no. S5886, Sigma) in DI water. The mixture was heated using a microwave to completely dissolve the agarose powder. The molten agarose solution was allowed to cool to ca. $60{ }^{\circ} \mathrm{C}$ before casting it into an inverse micromold (3D Petri Dish ${ }^{\mathrm{TM}}$, Microtissues Inc.) to form spherical microwells as illustrated in Figure S2 A I-II. After solidifying, the 3D Petri Dish with agarose was inverted and agarose microwells were extracted (Figure S2 A III-IV). The agarose microwells were equilibrated twice in Neurobasal medium at $37^{\circ} \mathrm{C}$ for $30 \mathrm{~min}$ in an incubator with $5 \% \mathrm{CO}_{2}$. The obtained cortical neurons were seeded in the microwells at a density of 500,000 cells/mold in $190 \mu \mathrm{L}$ of Neurobasal medium supplemented with $1 \times$ B27, $1 \%$ Glutamax and 1\% Penicillin/Streptomycin (Figure S2 B I-II). The cells settled in microwells by gravity (Figure S2 B III) and started to aggregate. An additional $2 \mathrm{~mL}$ of Neurobasal medium was added to the side of each well dropwise (Figure S2 B IV) to avoid disruption of the settled cells. $50 \%$ of the cell culture media was replaced with fresh media every 3 days. The cortical neurons self-assembled to form spheroids, which matured in culture for up to 30 days. Four-week-old cortical spheroids were harvested by inverting the micromold (Figure S2 B V). The harvested spheroids were placed in a Tyrode's solution-filled recording chamber attached to the 3D-SR-BA chip. 


\section{Electrical and optical recording from cortical spheroids}

Prior to recording, cortical spheroids were stained with $\mathrm{Ca}^{2+}$ indicator, Cal-520 (catalog no. 21130, AAT Bioquest). $5 \mu \mathrm{M}$ Cal-520 solution was prepared in Tyrode's solution. Tissues were incubated at $37{ }^{\circ} \mathrm{C}$ for $30 \mathrm{~min}$. Post incubation, the tissues were washed 3 times with $37{ }^{\circ} \mathrm{C}$ Tyrode's solution. Each 3D-SR-BA chip was glued to a printed circuit board (PCB) with soldered 36 pin connector (catalog no. A79024-001, Omnetics). The electrodes on the chip were wirebonded to the PCB using a manual wedge wire bonder (7476D Wire Bonder, West Bond). The chip was mounted on the microscope stage, and a $37^{\circ} \mathrm{C}$ temperature inside the measurement chamber was maintained using constant perfusion of heated (Inline heater, ThermoClamp, Automate Scientific) carbogen $\left(5 \% \mathrm{CO}_{2}\right.$ in $\mathrm{O}_{2}$ balance, Airgas) bubbled Tyrode's solution. $50 \mu \mathrm{M}$ glutamate solution was prepared in Tyrode's solution, with $\mathrm{pH}$ maintained via bubbling of carbogen gas.

For electrical recordings, the Omnetics connector on the printed circuit board was connected to a 32-channel amplifier (RHD2132, Intan Tech.), and the electrical signals were recorded using a commercially available acquisition system (RHD2000, Intan tech.) with a sampling rate of either $20 \mathrm{kHz}$ or $30 \mathrm{kHz}$. Fluorescence imaging was performed using an upright microscope equipped with a resonant confocal scanner (Nikon A1R) with a 20X/0.50 NA water immersion objective. All of the recordings were performed in a grounded Faraday cage.

Four-week old cortical spheroids were transferred to a 3D-SR-BA chip with a temperature controlled Tyrode's solution-filled chamber bubbled with carbogen gas. Using an $x-y-z$ micromanipulator (SMX, Sensapex), the arrays were unrolled and the spheroids were encapsulated by a 3D-SR-BA as illustrated by the schematic in Figure $1 \mathrm{~A}$ and the confocal microscopy image 
in Figure $1 \mathrm{C}$. Functional $\mathrm{Ca}^{2+}$ imaging was obtained by confocal microscopy from a single focal plane over a ca. $0.2 \mathrm{~mm}^{2}$ field of view, attained at a rate of 59 frames per second.

\section{Signal Processing and Data Analysis}

The recorded neural data from the 3D-SR-BAs was analyzed to eliminate electronic interference and identify single unit neural activity through spike-sorting. Second-order digital infinite impulse response (IIR) notch filters (bandwidth of $5 \mathrm{~Hz}$ ) were applied with a forwardbackward implementation to eliminate $60 \mathrm{~Hz}$ power-line interference signal and its harmonics. This postprocessing step was implemented using the open-source SciPy Python library (Python 3.7.2 and SciPy 1.2.1). ${ }^{21}$ Since the spectral content of the recorded neural signal is distributed over 300-7000 Hz frequency range, any localized narrow-band spectral feature can be attributed to unwanted interference, for example, from nearby electronic equipment or the power line. In our analysis, we detected narrow-band interference in the frequency domain by applying a peakdetection algorithm on the signal power spectral density (PSD). Peaks were identified from the peak-prominence, defined as the vertical distance between the peak and its lowest distinguishable contour line in the frequency domain. A peak-prominence threshold of 0.035 was empirically chosen to minimize distortion to the neural data while targeting prominent narrow-band interference. These identified frequency peaks were subsequently removed from the recorded data using the same IIR notch filter settings used to remove $60 \mathrm{~Hz}$ harmonics. Additionally, a bandpass filter from $300 \mathrm{~Hz}$ to $7 \mathrm{kHz}$ was applied using Python and the open-source SpikeInterface library to limit the spectral component of the signal to the spike band. ${ }^{22}$

Single unit neural activity was extracted from the filtered data using Python (SpikeInterface library) and the MountainSort4 spike-sorting algorithm. ${ }^{23}$ Spike events were first detected using a threshold of 5.0 standard deviations away from the mean of the recorded signal. Dimensionality 
reduction was performed on the events using principle component analysis (PCA) with $n=3$ components on each recording channel. Then, the detected events were clustered into separate neural units based on the PCA results using the MountainSort4 algorithm. ${ }^{24}$ The neural unit clusters were manually curated by inspecting the average spike waveforms and inter-spike intervals for each unit cluster to remove noise and recording artifacts misidentified as neural units. Firing rate histograms were calculated from the spike event time information using a bin size of 2 seconds.

\section{Results and Discussion}

Fabrication and Electrochemical Characterization of Biosensors on 3D-SR-BA.

3D-SR-BA is realized using a pre-stressed metal/polymer multilayer structure as previously reported. ${ }^{15}$ Our self-rolling platform is fabricated on a planar surface (Figure 1 A I, II) on top of a sacrificial layer and can accommodate multiple sensor types. ${ }^{15}$ Upon dissolution of the Ge sacrificial layer in $1 \%$ hydrogen peroxide, the pre-stress in the metallic traces guides the array's self-rolling, achieving a controlled 3D sensor configuration with a desired curvature (Figure 1 A I, II, and Figure S3, Materials and Methods). ${ }^{15}$ The curvature of the 3D-SR-BAs is controlled by modulating the polymer insulation layer thickness, resulting in highly-tunable arrays with controllable radius of curvature. ${ }^{15}$ In this study, we fabricated the 3D-SR-BA (radius of curvature $96 \pm 4 \mu \mathrm{m}$, Figure $1 \mathrm{~B}$, Figure S3) to match the dimensions of a cortical spheroid (diameter 202 $\pm 24 \mu \mathrm{m}$, Figure $1 \mathrm{C}$, Figure S4). 


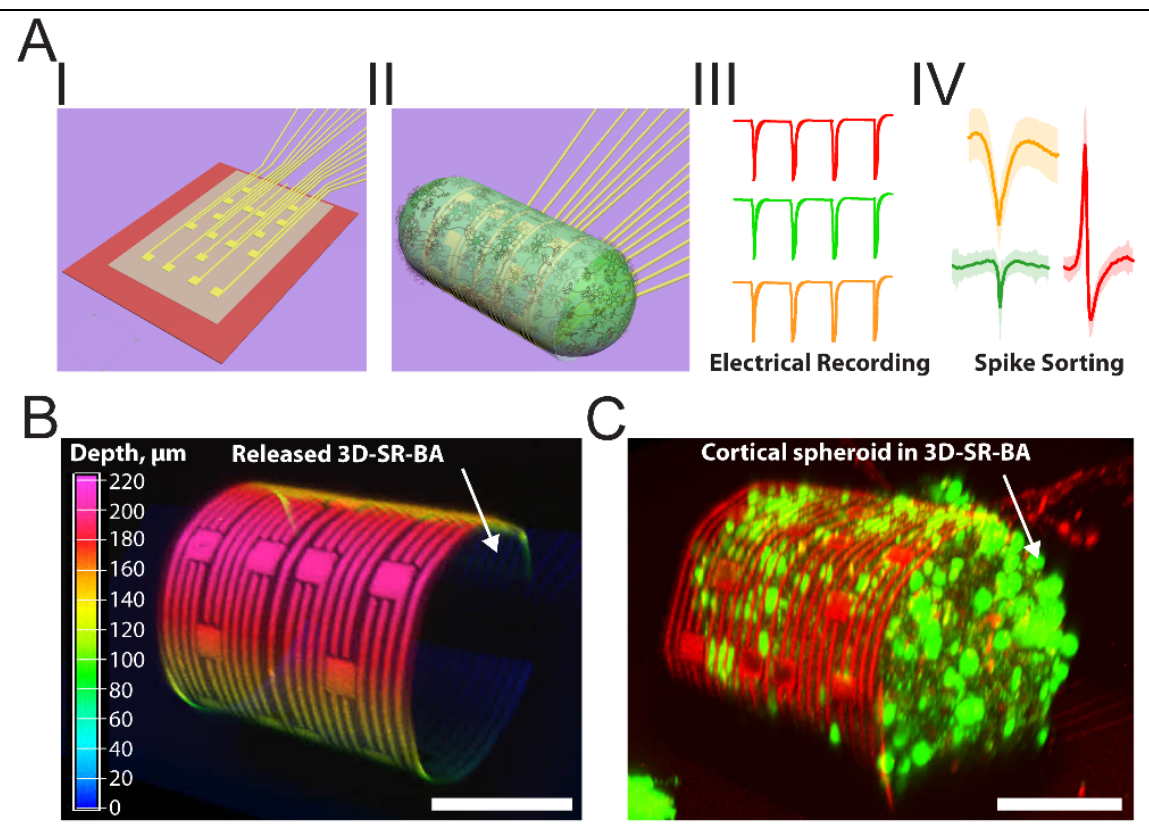

Figure 1. Three-dimensional (3D) self-rolled biosensor array (3D-SR-BA) for 3D recording from cortical spheroids. (A) A schematic of the experiment: (I) The 3D-SR-BA is released to conform in $3 \mathrm{D}$, (II) A cortical spheroid is encapsulated in the 3D-SR-BAs, and (III) The electrical recording is obtained. (IV) The recorded data is processed, and spike-sorting algorithms are used to identify individual neuronal activity. (B) A 3D confocal microscopy image of 3D-SR-BA. Color bar represents the depth in $\mu \mathrm{m}$. (C) A 3D confocal microscopy image of cortical spheroid labeled with $\mathrm{Ca}^{2+}$ indicator dye (Cal-520, green fluorescence) encapsulated by the 3D-SR-BA. Scale bars are $100 \mu \mathrm{m}$.

Microfabricated biosensors, such as MEAs, are used extensively in neural interfaces ${ }^{25}$ due to their potential for long-term, multisite, and simultaneous recordings with high temporal resolution. ${ }^{26}$ Microfabrication techniques allow tailoring the array geometry, size and substrate mechanical properties (e.g. fabrication of ultra-compliant $\operatorname{probes}^{25,27-31}$ ). In this study, seventeen $25 \mu \mathrm{m} \times 25 \mu \mathrm{m}$ microelectrodes are fabricated on the $235 \mu \mathrm{m} \times 530 \mu \mathrm{m}$ 3D-SR-BA (Materials and Methods) and assembled in 3D to interrogate the electrical activity of cortical spheroids with cellular resolution, as illustrated by the 3D confocal image (Figure $1 \mathrm{~B}$ ). To reduce impedance and produce higher signal amplitudes during recording, ${ }^{18}$ the Au microelectrodes are coated with with PEDOT:PSS via electrodeposition post fabrication. ${ }^{18}$ PEDOT:PSS modification resulted in a reduction in microelectrode impedance from $1.43 \pm 0.40 \mathrm{M} \Omega$ prior to modification (Figure S5, 
blue trace, measured at $1 \mathrm{kHz}, \mathrm{n}=11$ ) to $21.3 \pm 3.5 \mathrm{k} \Omega$ after electropolymerization (Figure S5, orange trace, measured at $1 \mathrm{kHz}, \mathrm{n}=11) .{ }^{15,18}$ The reduced impedance results from improved capacitance due to the electrical double layer formation at the interconnected PEDOT-rich and PSS-rich grains. ${ }^{32}$

Electrical and Optical Recording of Spontaneous Firing of Neurons in Cortical Spheroids

3D electrophysiological signals were recorded from cortical spheroids using 3D-SR-BAs. Cortical spheroids were compacted in agarose microwells for 28 days. Rod-shaped cortical tissues were transferred to the 3D-SR-BA chip with a temperature-controlled carbogenated Tyrode's solution-filled chamber (Materials and Methods). Using an x-y-z micromanipulator, the 3D-SRBAs were unrolled allowing each array to encapsulate a single spheroid as illustrated in Figure 2 A. Six microelectrodes arranged in 3D (Figure 2 B) simultaneously recorded the field potentials from the encapsulated cortical spheroid (Spheroid 1). The 3D-SR-BA electrical recordings were obtained concurrently with functional $\mathrm{Ca}^{2+}$ imaging (Figure $2 \mathrm{C}$, Supplementary Movie 1 ). $\mathrm{Ca}^{2+}$ ions flow across the cell membrane during an action potential, allowing optical monitoring of the resulting electrical activity via fluorescence imaging. ${ }^{33}$ Functional $\mathrm{Ca}^{2+}$ transients (Figure $2 \mathrm{~A}$, green fluorescence, Figure 2 C, Supplementary Movie 1) were simultaneously imaged from a single focal plane over a ca. $0.2 \mathrm{~mm}^{2}$ field of view. The spheroid was stained with $\mathrm{Ca}^{2+}$ indicator (Cal-520, Materials and Methods). Cortical neurons cultured in vitro for an extended time exhibit synchronized neural activity. ${ }^{34}$ This result was confirmed by comparing the functional $\mathrm{Ca}^{2+}$ transients between individual region of interest (ROI) and the whole field of view (Figure S6). The $\mathrm{Ca}^{2+}$ transients acquired from the whole field of view captures all the transients from the ROIs. 


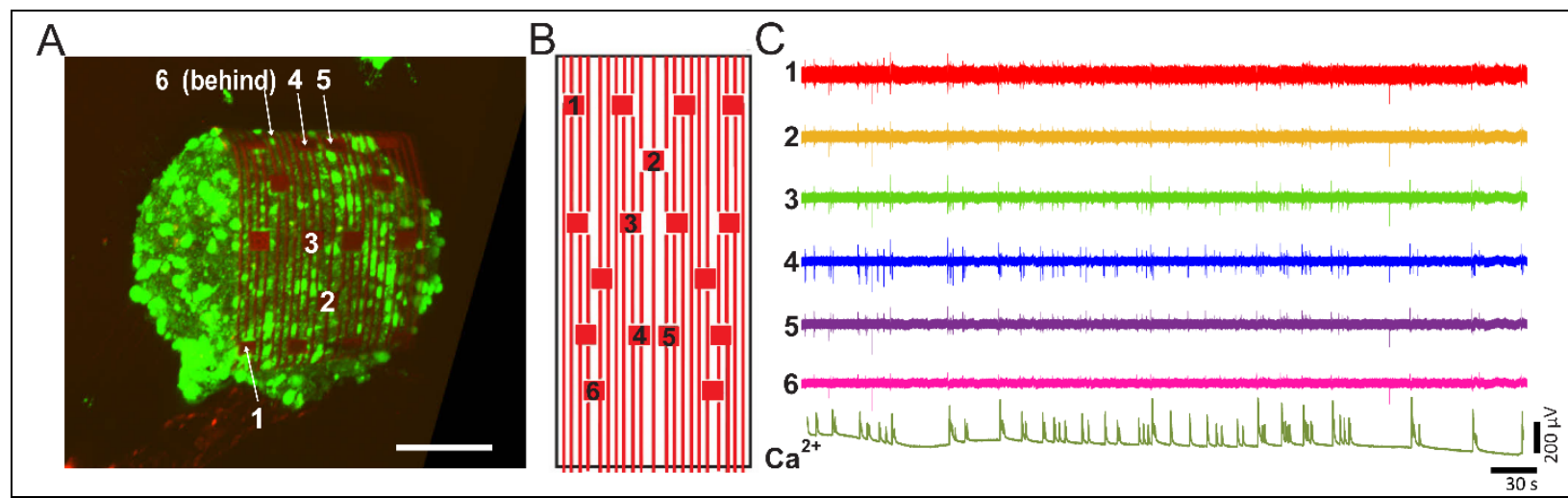

Figure 2. Electrical and optical recordings in 3D of cortical spheroids using 3D self-rolled biosensor array (3D-SR-BA). (A) A 3D confocal microscopy image of 3D cortical spheroid labeled with $\mathrm{Ca}^{2+}$ indicator dye (Cal-520, green fluorescence) encapsulated by the 3D-SR-BA. Scale bar is $100 \mu \mathrm{m}$. (B) A 2D map of the recording microelectrodes labeled in panel (A). (C) Representative voltage traces recorded from spontaneously firing cortical neurons via channels labeled in panel (A) and (B) with simultaneously recorded $\mathrm{Ca}^{2+}$ fluorescence intensity (whole tissue).

The raw neural data recorded from six electrodes was analyzed (Materials and Methods) and five distinct single unit spikes were identified in cortical Spheroid 1. The averaged waveforms of all sorted spikes associated with each unit are illustrated in Figure 3 A (solid color traces). The standard deviations of individual spike events in each unit are represented by the shaded regions in Figure 3 A. Different unit waveforms are detected by different microelectrodes near the firing neurons in the neural spheroid. The corresponding electrode is identified by a number on the left of each waveform plot in Figure 3 A. Three out of the five units were primarily detected on individual electrode channels: each unit on individual channel, indicating that the responsible neurons were close to each of those electrodes. Units 3 and 4 were detected on both channels 4 and 5 (Figure $3 \mathrm{~A}$ ), indicating that the firing neurons were located between those electrodes (Figure 2 A, B). 


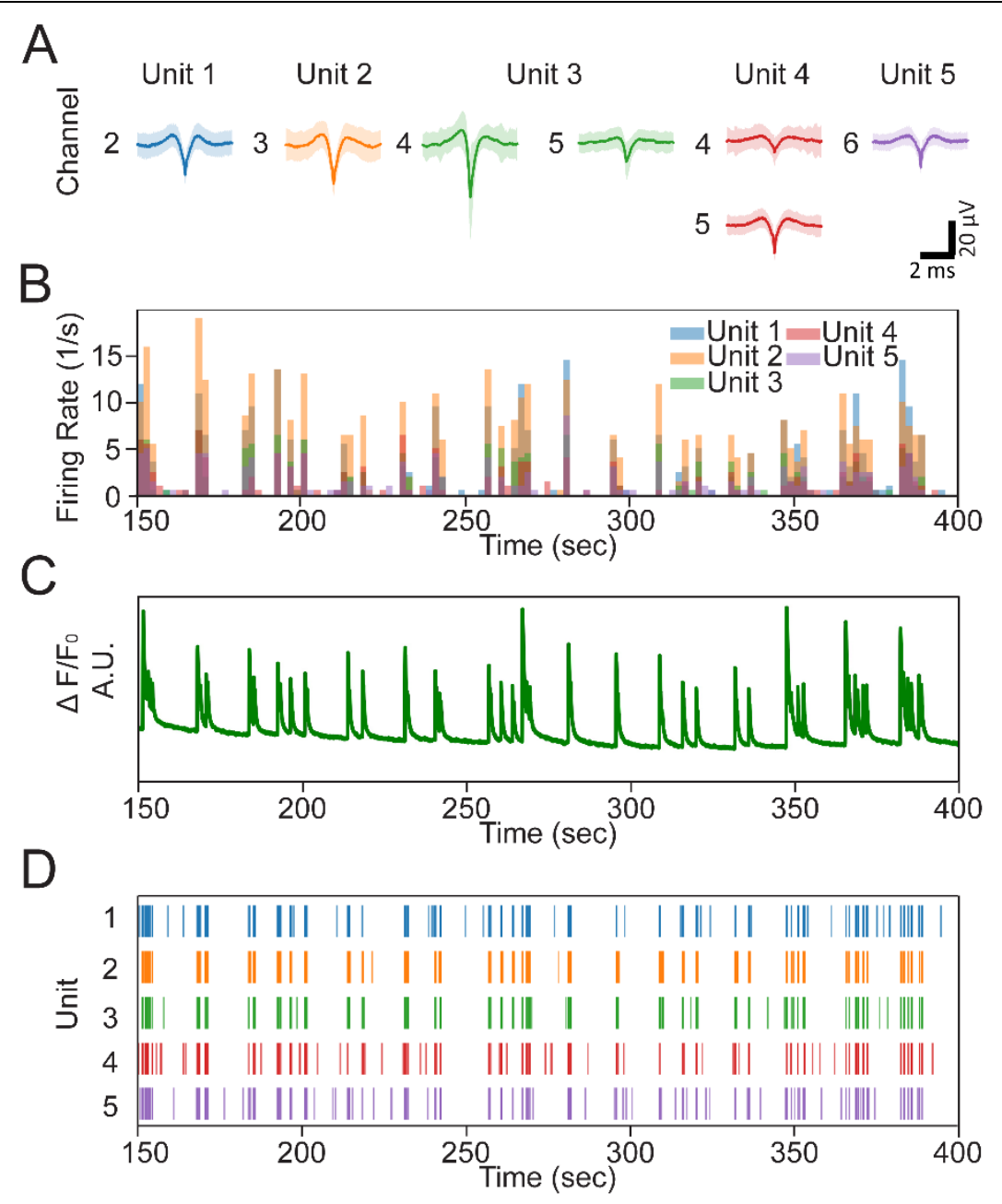

Figure 3. Spontaneous cortical neuron activity correlated to $\mathrm{Ca}^{2+}$ fluorescence transients. (A) Averaged spike (solid trace) and \pm standard deviation (shaded region) detected in each unit. The number on the left of the trace is the channel where the unit was detected. (B) Overlaid firing rate of representative units. (C) Simultaneously recorded $\mathrm{Ca}^{2+}$ fluorescence intensity. (D) Raster plots of the firing patterns of the detected units.

The firing rates of individual spike-sorted neural units (Figure 3 B and Figure S7 A) indicated bursts of activity up to 20 spikes/s, which corresponds well with the fluorescent signal of the functional $\mathrm{Ca}^{2+}$ imaging (Figure $3 \mathrm{C}$, Figure S7 B, Supplementary Movie 1). The precise spike timings are shown in the raster plot in Figure $3 \mathrm{D}$. It is evident that the firing patterns of the detected units are synchronized across the spheroid, which agrees with previously published studies on synchronization of cortical neuron clusters cultured in vitro. ${ }^{34}$ This synchrony is also observed in the $\mathrm{Ca}^{2+}$ transients obtained from different regions of the spheroid (Figure S6). 


\section{Electrical and Optical Recording of glutamate-induced firing of neurons in cortical spheroids}

As a proof-of-concept for 3D-SR-BA use in drug effect monitoring, the effect of glutamate, the main excitatory neurotransmitter of the nervous system, ${ }^{35}$ was investigated. The effect of glutamate on glutamatergic neurons is well established. ${ }^{35}$ The 3D-SR-BA was used to continuously record ephys activity while glutamate solution $(50 \mu \mathrm{M})$ was perfused into a chamber containing the encapsulated spheroid. Simultaneous functional $\mathrm{Ca}^{2+}$ imaging was used to supplement the ephys recording and illustrated the same cellular activity (Figure 4 A, Supplementary Movie 2, Materials and Methods). The same five neuronal units isolated during spontaneous neural activity recording (Figure $4 \mathrm{~B}$ ) were detected by the data analysis pipeline. Both the electrical and optical recordings show elevated neuronal firing rates following the introduction of glutamate (Figure 4 A, C, D, Figure S8, and Supplementary Movie 2). The increased firing rate is the expected after the addition of excitatory neurotransmitter glutamate. ${ }^{35}$ We note that the time delay between the onset and offset of glutamate flow and the change in neuronal firing rate is due to the time required for glutamate to perfuse into the recording chamber. The raster plots of the detected units illustrate a firing rate increase between 4.5-fold and 9.7-fold for individual units (above the spontaneous activity baseline level) upon addition of glutamate (Figure 4 E, Figure S8).

The 3D recording demonstrated in this work was repeated by measuring the spontaneous and glutamate-induced firing from three independent neural spheroids (Technical Note 1 in Supplementary Information). The change in spike counts upon glutamate addition are summarized for each spheroid in Figure S9. A notable increase in spiking activity of individual units within a spheroid upon glutamate addition was observed (4.5-9.7-fold, 6.1-14.5-fold, and 2.5-37.9-fold for units in Spheroid 1, 2 and 3, respectively). This increase is associated with the glutamate flow indicated by the green-shaded regions in Figure S9. For each spheroid, we introduced glutamate 
in multiple cycles and after each cycle glutamate was washed from the chamber. The change in neuronal firing rate (increase upon addition and decrease upon removal) consistently followed the glutamate cycles. The variation of neuronal response to glutamate among different units in each spheroid, characterized as the change in firing rates, can be attributed to biological variability in the spontaneous activity of each unit: neurons that fire more sparsely under the spontaneous condition illustrate a more pronounced increase under the glutamate-perfused condition. 


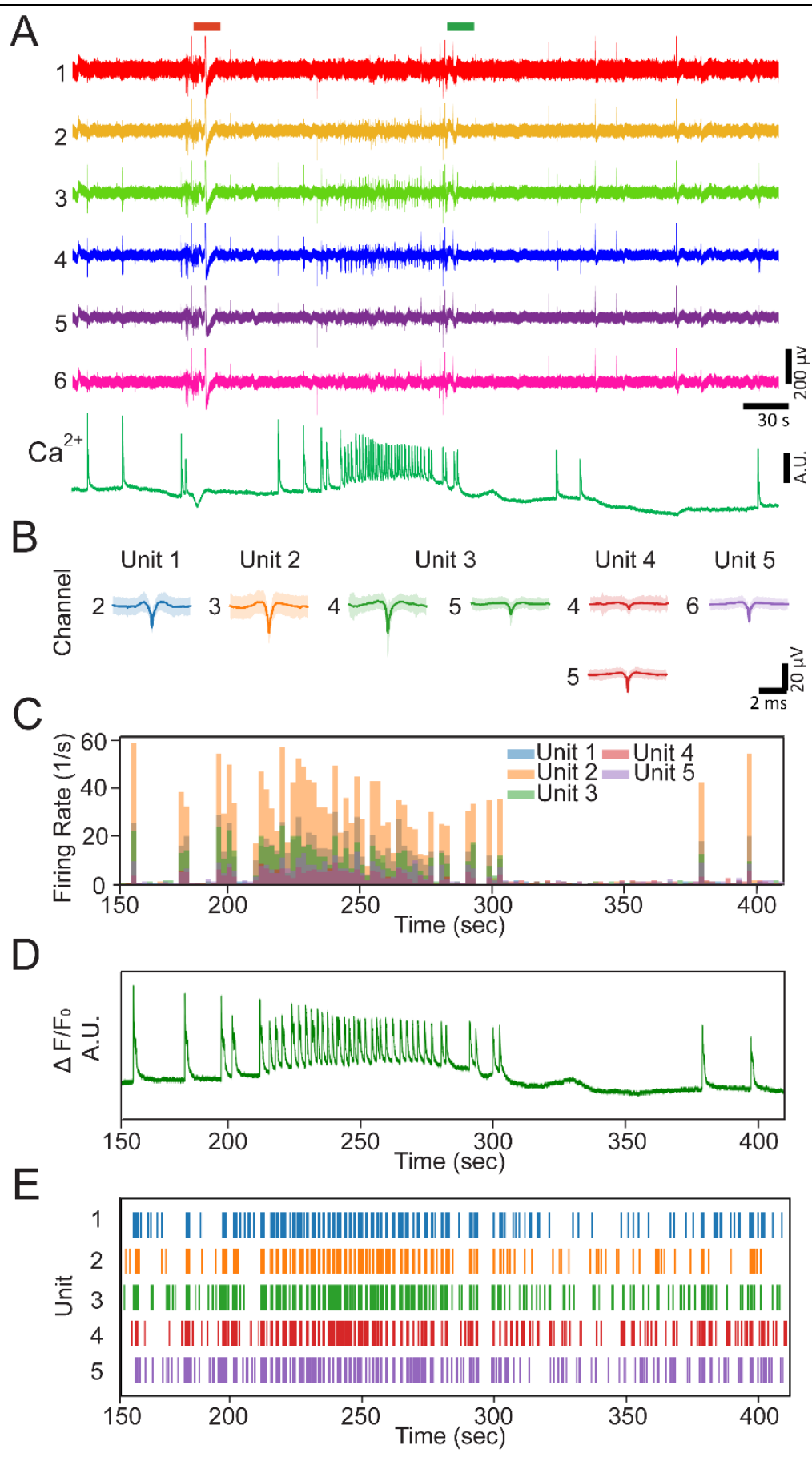

Figure 4. Glutamate-induced activity of cortical neuron is correlated to $\mathrm{Ca}^{2+}$ fluorescence transients. (A) Representative ephys recording of glutamate-induced cortical neuron activity and corresponding $\mathrm{Ca}^{2+}$ fluorescence transients. (B) Averaged spike (solid trace) and \pm standard deviation (shaded region) detected in each unit. The number on the left of the trace is the channel where the unit was detected. (C) Overlaid firing rate of representative units. (D) Simultaneously recorded $\mathrm{Ca}^{2+}$ fluorescence intensity. (E) Raster plots of the firing patterns of the detected units. 


\section{Conclusion and future directions}

This work demonstrates the first 3D ephys recording from a neuronal spheroid using a 3D biosensor platform. The novel architecture of the 3D-SR-BA allows for high-resolution recordings of neural activity currently unattainable by other recording methods. It provides a robust, tissue incorporated, continuous, sensitive, and reproducible electrical recordings. Integrating a signalprocessing and spike sorting analysis pipeline enables isolation of individual distinct neural units from the 3D-SR-BA recorded data. The electrical recordings were supplemented with functional $\mathrm{Ca}^{2+}$ imaging and firing rates of the individual units detected with the 3D-SR-BA corresponded to the $\mathrm{Ca}^{2+}$ transients imaged optically. Using our 3D-SR-BA neural spheroid system, we are able to measure the spheroid's response to glutamate addition which results in increased neuronal activity as indicated by the difference in the firing pattern compared to the spontaneous activity recording. The ability to record complex neural electrical activity using 3D-SR-BA opens a plethora of possibilities for longer-term study of 3D neuronal tissues. The approach presented in this work can enable studies of neuronal functions in 3D, e.g. investigation of brain organoid development and functionality in both healthy and disease models. ${ }^{12}$ Moreover, our approach can assist with the discovery of new therapeutics for neurological disorders using an in vitro model which is a better approximation of in vivo conditions. 


\section{Acknowledgments}

T.C.-K. acknowledges funding support from the National Science Foundation (NSF) under Award CBET1552833, the Office of Naval Research under Award N000141712368, and the Defense Advanced Research Projects Agency under Award AWD00001593 (416052-5). M.C. acknowledges funding support from the NSF under Award NCS-FO1926804. A.K. acknowledges support from the Neil and Jo Bushnell Fellowship in Engineering. We also acknowledge support from the Department of Materials Science and Engineering Materials Characterization Facility supported by Grant MCF-677785. J.W.R. acknowledges support by the Carnegie Mellon University Ben Cook Presidential Graduate Fellowship, the Carnegie Mellon University Richard King Mellon Foundation Presidential Fellowship in the Life Sciences, the Axel Berny Presidential Graduate Fellowship, and Philip and Marsha Dowd. 


\section{References:}

1. Takahashi, T., Organoids for drug discovery and personalized medicine. Annual review of pharmacology and toxicology 2019, 59, 447-462.

2. Yu, F.; Hunziker, W.; Choudhury, D., Engineering microfluidic organoid-on-a-chip platforms. Micromachines 2019, 10 (3), 165.

3. Dutta, D.; Heo, I.; Clevers, H., Disease modeling in stem cell-derived 3D organoid systems. Trends in molecular medicine 2017, 23 (5), 393-410.

4. Park, J.; Wetzel, I.; Marriott, I.; Dréau, D.; D’Avanzo, C.; Kim, D. Y.; Tanzi, R. E.; Cho, H., A 3D human triculture system modeling neurodegeneration and neuroinflammation in Alzheimer's disease. Nature neuroscience 2018, 21 (7), 941-951.

5. Quadrato, G.; Brown, J.; Arlotta, P., The promises and challenges of human brain organoids as models of neuropsychiatric disease. Nature medicine 2016, 22 (11), 1220.

6. Lancaster, M. A.; Renner, M.; Martin, C.-A.; Wenzel, D.; Bicknell, L. S.; Hurles, M. E.; Homfray, T.; Penninger, J. M.; Jackson, A. P.; Knoblich, J. A., Cerebral organoids model human brain development and microcephaly. Nature 2013, 501 (7467), 373.

7. Zhang, B.; Korolj, A.; Lai, B. F. L.; Radisic, M., Advances in organ-on-a-chip engineering. Nature Reviews Materials 2018, 3 (8), 257-278.

8. Pampaloni, F.; Reynaud, E. G.; Stelzer, E. H., The third dimension bridges the gap between cell culture and live tissue. Nature reviews Molecular cell biology 2007, 8 (10), 839-845.

9. Qian, X.; Nguyen, H. N.; Song, M. M.; Hadiono, C.; Ogden, S. C.; Hammack, C.; Yao, B.; Hamersky, G. R.; Jacob, F.; Zhong, C., Brain-region-specific organoids using mini-bioreactors for modeling ZIKV exposure. Cell 2016, 165 (5), 1238-1254.

10. Hopkins, A. M.; DeSimone, E.; Chwalek, K.; Kaplan, D. L., 3D in vitro modeling of the central nervous system. Progress in neurobiology 2015, 125, 1-25.

11. Quadrato, G.; Nguyen, T.; Macosko, E. Z.; Sherwood, J. L.; Yang, S. M.; Berger, D. R.; Maria, N.; Scholvin, J.; Goldman, M.; Kinney, J. P., Cell diversity and network dynamics in photosensitive human brain organoids. Nature 2017, 545 (7652), 48-53.

12. Trujillo, C. A.; Gao, R.; Negraes, P. D.; Gu, J.; Buchanan, J.; Preissl, S.; Wang, A.; Wu, W.; Haddad, G. G.; Chaim, I. A., Complex oscillatory waves emerging from cortical organoids model early human brain network development. Cell stem cell 2019, 25 (4), 558-569. e7.

13. McDonald, M.; Sebinger, D.; Brauns, L.; Gonzalez-Cano, L.; Menuchin-Lasowski, Y.; Psathaki, O.-E.; Stumpf, A.; Rauen, T.; Schöler, H.; Jones, P. D., A mesh microelectrode array for non-invasive electrophysiology within neural organoids. bioRxiv 2020.

14. Pachitariu, M.; Stringer, C.; Harris, K. D., Robustness of spike deconvolution for neuronal calcium imaging. Journal of Neuroscience 2018, 38 (37), 7976-7985.

15. Kalmykov, A.; Huang, C.; Bliley, J.; Shiwarski, D.; Tashman, J.; Abdullah, A.; Rastogi, S. K.; Shukla, S.; Mataev, E.; Feinberg, A. W., Organ-on-e-chip: Three-dimensional self-rolled biosensor array for electrical interrogations of human electrogenic spheroids. Science advances 2019, 5 (8), eaax0729.

16. Cohen-Karni, T.; Timko, B. P.; Weiss, L. E.; Lieber, C. M., Flexible electrical recording from cells using nanowire transistor arrays. Proceedings of the National Academy of Sciences 2009, 106 (18), 7309-7313.

17. Rastogi, S. K.; Bliley, J.; Shiwarski, D. J.; Raghavan, G.; Feinberg, A. W.; Cohen-Karni, T., Graphene Microelectrode Arrays for Electrical and Optical Measurements of Human Stem Cell-Derived Cardiomyocytes. Cellular and Molecular Bioengineering 2018, 1-12. 
18. Cui, X.; Martin, D. C., Electrochemical deposition and characterization of poly (3, 4ethylenedioxythiophene) on neural microelectrode arrays. Sensors and Actuators B: Chemical 2003, 89 (1-2), 92-102.

19. Dingle, Y.-T. L.; Boutin, M. E.; Chirila, A. M.; Livi, L. L.; Labriola, N. R.; Jakubek, L. M.; Morgan, J. R.; Darling, E. M.; Kauer, J. A.; Hoffman-Kim, D., Three-dimensional neural spheroid culture: an in vitro model for cortical studies. Tissue Engineering Part C: Methods 2015, 21 (12), 1274-1283.

20. Napolitano, A. P.; Dean, D. M.; Man, A. J.; Youssef, J.; Ho, D. N.; Rago, A. P.; Lech, M. P.; Morgan, J. R., Scaffold-free three-dimensional cell culture utilizing micromolded nonadhesive hydrogels. Biotechniques 2007, 43 (4), 494-500.

21. Virtanen, P.; Gommers, R.; Oliphant, T. E.; Haberland, M.; Reddy, T.; Cournapeau, D.; Burovski, E.; Peterson, P.; Weckesser, W.; Bright, J., SciPy 1.0: fundamental algorithms for scientific computing in Python. Nature methods 2020, 17 (3), 261-272.

22. Buccino, A. P.; Hurwitz, C. L.; Magland, J.; Garcia, S.; Siegle, J. H.; Hurwitz, R.; Hennig, M. H., SpikeInterface, a unified framework for spike sorting. BioRxiv 2019, 796599.

23. Chung, J. E.; Magland, J. F.; Barnett, A. H.; Tolosa, V. M.; Tooker, A. C.; Lee, K. Y.; Shah, K. G.; Felix, S. H.; Frank, L. M.; Greengard, L. F., A fully automated approach to spike sorting. Neuron 2017, 95 (6), 1381-1394. e6.

24. Rey, H. G.; Pedreira, C.; Quiroga, R. Q., Past, present and future of spike sorting techniques. Brain research bulletin 2015, 119, 106-117.

25. Lacour, S. P.; Benmerah, S.; Tarte, E.; FitzGerald, J.; Serra, J.; McMahon, S.; Fawcett, J.; Graudejus, O.; Yu, Z.; Morrison, B., Flexible and stretchable micro-electrodes for in vitro and in vivo neural interfaces. Medical \& biological engineering \& computing 2010, 48 (10), 945954.

26. Rastogi, S. K.; Kalmykov, A.; Johnson, N.; Cohen-Karni, T., Bioelectronics with nanocarbons. Journal of Materials Chemistry B 2018, 6 (44), 7159-7178.

27. Hong, G.; Yang, X.; Zhou, T.; Lieber, C. M., Mesh electronics: a new paradigm for tissue-like brain probes. Current opinion in neurobiology 2018, 50, 33-41.

28. Hong, G.; Viveros, R. D.; Zwang, T. J.; Yang, X.; Lieber, C. M., Tissue-like neural probes for understanding and modulating the brain. Biochemistry 2018, 57 (27), 3995-4004.

29. Zhou, T.; Hong, G.; Fu, T.-M.; Yang, X.; Schuhmann, T. G.; Viveros, R. D.; Lieber, C. M., Syringe-injectable mesh electronics integrate seamlessly with minimal chronic immune response in the brain. Proceedings of the National Academy of Sciences 2017, 114 (23), 58945899.

30. Luan, L.; Wei, X.; Zhao, Z.; Siegel, J. J.; Potnis, O.; Tuppen, C. A.; Lin, S.; Kazmi, S.; Fowler, R. A.; Holloway, S., Ultraflexible nanoelectronic probes form reliable, glial scar-free neural integration. Science advances 2017, 3 (2), e1601966.

31. Yang, X.; Zhou, T.; Zwang, T. J.; Hong, G.; Zhao, Y.; Viveros, R. D.; Fu, T.-M.; Gao, T.; Lieber, C. M., Bioinspired neuron-like electronics. Nature materials 2019, 18 (5), 510-517.

32. Volkov, A. V.; Wijeratne, K.; Mitraka, E.; Ail, U.; Zhao, D.; Tybrandt, K.; Andreasen, J. W.; Berggren, M.; Crispin, X.; Zozoulenko, I. V., Understanding the capacitance of PEDOT: PSS. Advanced Functional Materials 2017, 27 (28), 1700329.

33. Akerboom, J.; Chen, T.-W.; Wardill, T. J.; Tian, L.; Marvin, J. S.; Mutlu, S.; Calderón, N. C.; Esposti, F.; Borghuis, B. G.; Sun, X. R., Optimization of a GCaMP calcium indicator for neural activity imaging. Journal of neuroscience 2012, 32 (40), 13819-13840. 
34. Murphy, T. H.; Blatter, L. A.; Wier, W. G.; Baraban, J. M., Spontaneous synchronous synaptic calcium transients in cultured cortical neurons. Journal of Neuroscience 1992, 12 (12), 48344845.

35. Zhou, Y.; Danbolt, N., Glutamate as a neurotransmitter in the healthy brain. Journal of neural transmission 2014, 121 (8), 799-817.

36. Aebersold, M. J.; Dermutz, H.; Demkó, L.; Cogollo, J. F. S.; Lin, S.-C.; Burchert, C.; Schneider, M.; Ling, D.; Forró, C.; Han, H.; Zambelli, T.; Vörös, J., Local Chemical Stimulation of Neurons with the Fluidic Force Microscope (FluidFM). ChemPhysChem 2018, 19 (10), 1234-1244. 
Supplementary Information for:

\section{Bioelectrical Interfaces with Cortical Spheroids in Three-Dimensions}

Anna Kalmykov ${ }^{1, \S}$, Jay W. Reddy ${ }^{2, \S}$, Esther Bedoyan ${ }^{2, \S}$, Yingqiao Wang ${ }^{3}$, Raghav Garg ${ }^{3}$, Sahil K. Rastogi ${ }^{1}$, Devora Cohen-Karni ${ }^{4}$, Maysamreza Chamanzar ${ }^{2, *}$, Tzahi Cohen-Karni ${ }^{1,3, *}$

${ }^{1}$ Department of Biomedical Engineering, Carnegie Mellon University, Pittsburgh, PA- 15213, USA.

${ }^{2}$ Department of Electrical and Computer Engineering, Carnegie Mellon University, Pittsburgh, PA- 15213, USA.

${ }^{3}$ Department of Materials Science and Engineering, Carnegie Mellon University, Pittsburgh, PA15213, USA.

${ }^{4}$ Preclinical education, Lake Erie College of Osteopathic Medicine at Seton Hill, Greensburg, PA15601, USA.

$\S$ These authors contributed equally to this work.

* Corresponding authors: Tzahi Cohen-Karni tzahi@andrew.cmu.edu; Maysamreza Chamanzar mchamanz@andrew.cmu.edu

This file includes:

Figures S1-S19

Technical note 1: Experimental Replication in Additional Cortical Spheroids

Supplementary Movies 1-6 


\section{Technical Note 1: Experimental Replication in Additional Cortical Spheroids}

The 3D ephys recording and functional $\mathrm{Ca}^{2+}$ imaging demonstrated in this work was repeated under spontaneous and glutamate-perfused conditions for three separately prepared cortical spheroids.

\section{Spheroid 2}

A second spheroid stained with a $\mathrm{Ca}^{2+}$ indicator (green fluorescence) was encapsulated by the 3D-SR-BA (Figure S10 A). The recording channel locations are identified by the labels in Figure S10 A, B. The recorded voltage traces are shown alongside the $\mathrm{Ca}^{2+}$ fluorescence intensity versus time for the spontaneous (Figure S10 C, Supplementary Movie 3) and glutamate-perfused (Figure S10 D, Supplementary Movie 4) conditions. This recording had several pronounced artifacts, potentially emerging from the initiation of the glutamate flow or other environmental noise (marked by asterisks on Figures S10 C, D). The addition of glutamate (green bars, Figure S10 D) produces a notable increase in ephys and $\mathrm{Ca}^{2+}$ activity. This cortical spheroid was subjected to three glutamate addition/wash-out cycles, demonstrating a repeatable glutamate response.

Spike-sorting of the recorded spontaneous ephys activity from this spheroid revealed three distinctly clustered units (Figure S11 A). The firing rates of these units illustrated on Figure S11 $\mathrm{B}$ and Figure S12 A, are supplemented by the simultaneously recorded functional $\mathrm{Ca}^{2+}$ imaging (Figure S11 C, Figure S12 B, Supplementary Movie 3). The spontaneous firing is sparse for this neuronal construct, shown in the raster plots for individual units (Figure S11 D). Upon glutamate addition, the number of active detected units in the second spheroid increases to eight (Figure S13 A). This potentially indicates glutamate stimulation of glutamatergic cortical neurons that are otherwise dormant. ${ }^{36}$ Several units were identified across multiple channels, such as units 5 and 7 , suggesting that they are located between the two electrodes. The glutamate addition resulted in 
increased spiking activity, as evidenced by the increased firing rates (Figure S13 B, Figure S14 A, and Supplementary Movie 4), which reverted upon glutamate removal. The onset of $\mathrm{Ca}^{2+}$ transients aligns with the firing of individual units (Figure S13 B, C, D and Figure S14). The introduction of glutamate induced a 6.1-fold increase to 14.5 -fold increase in the firing rate of individual neurons.

\section{Spheroid 3}

Using the same method, the third spheroid was labeled with a $\mathrm{Ca}^{2+}$ indicator and encapsulated by the 3D-SR-BA (Figure S15 A). The electrical activity of this spheroid was recorded by the channels marked in Figure S15 A, B. The ephys recordings and functional $\mathrm{Ca}^{2+}$ imaging were obtained for the spontaneous (Figure S15 C, Supplementary Movie 5) and the glutamate-induced neuronal activity (Figure S15 D, Supplementary Movie 6). An artifact, potentially from the initiation of the glutamate flow, is marked by an asterisk on Figure S15 D. The neural activity measured by ephys recordings and functional $\mathrm{Ca}^{2+}$ imaging increased after glutamate addition and decreased upon glutamate removal. This spheroid was subjected to two glutamate addition and removal cycles, demonstrating the expected glutamate response in each cycle.

Six distinctly clustered units were identified in the spontaneous recording (Figure S16 A). The firing rates of these units illustrated in Figure S16 B and Figure S17 A, are supplemented by the simultaneously recorded functional $\mathrm{Ca}^{2+}$ imaging (Figure S16 C, D, Figure S17 B, and Supplementary Movie 5). Units 3 and 4 were identified across multiple channels, suggesting that they are positioned between those electrodes. Upon glutamate addition, however, the number of detected units in spheroid 3 decreases to four (Figure S18 A). Several units were still identified across multiple channels, such as units 2 and 4, suggesting that the responsible neuron is located between the electrodes. The glutamate addition resulted in increased spiking activity, as evidenced by the increased firing rates (Figure S18 B, Figure S19 A, and Supplementary Movie 6), which 
reverted upon glutamate removal. The onset of $\mathrm{Ca}^{2+}$ transients aligns with increased firing of individual units (Figure S18 B, C, D and Figure S19). The introduction of glutamate induced an increase between 2.5-fold and 37.9-fold in firing rate of individual neurons within the spheroid.

\section{Results Across Multiple Spheroids}

The data indicate that in some cases (as in Spheroid 2), the number of detected units is higher after the introduction of glutamate, whereas in others (as in Spheroid 3), fewer units are detected in the glutamate condition. The addition of glutamate could excite different subsets of glutamatergic neurons which only partially overlaps with the population of spontaneously active neurons. Alternatively, the detection of specific units could also be an artifact of the spike-sorting, whereby the varied distribution of neural activity under glutamate stimulation lends itself to better or poorer single-unit isolation for certain neurons.

The change in spike counts are summarized for each condition in Figure S9, illustrating a notable increase in spike count from spontaneous to glutamate-induced firing in each of the recordings. Such an increase is evidently associated with the glutamate flow specified by the greenshaded regions in Figure S9. The notable increase of neuronal firing upon multiple cycles of glutamate addition and decrease in firing upon glutamate removal indicates a predicted and repeatable response in this biological system. 


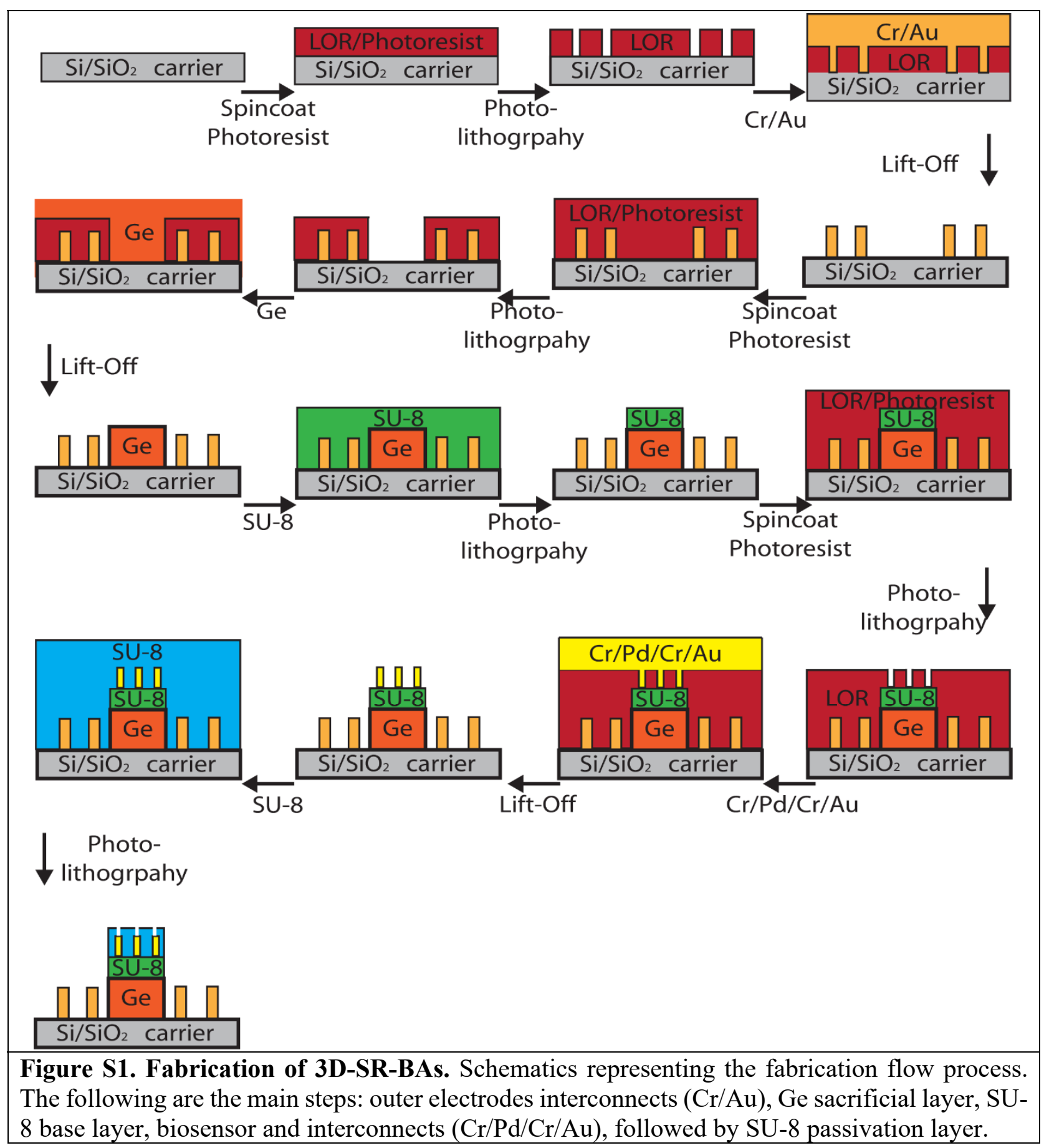




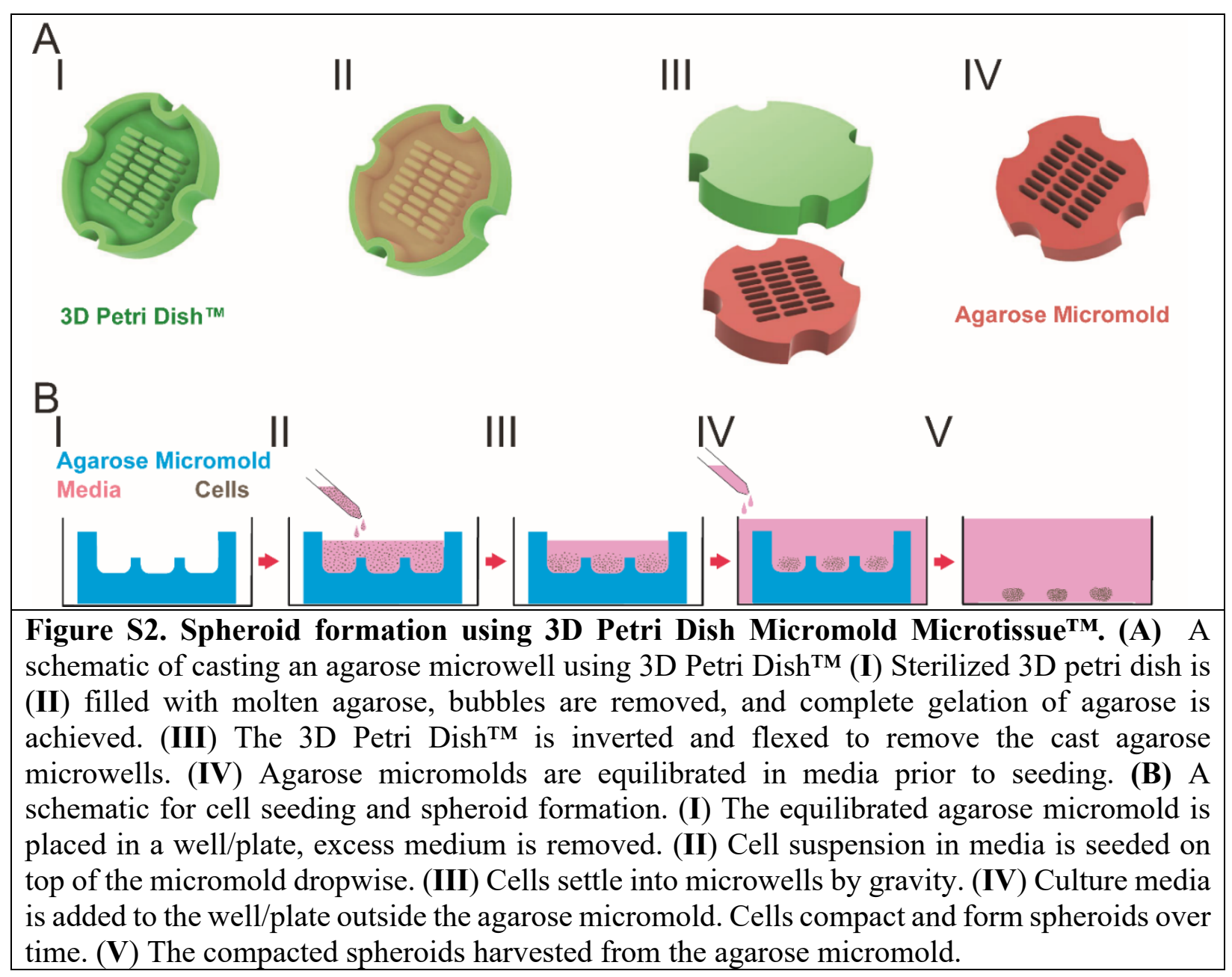




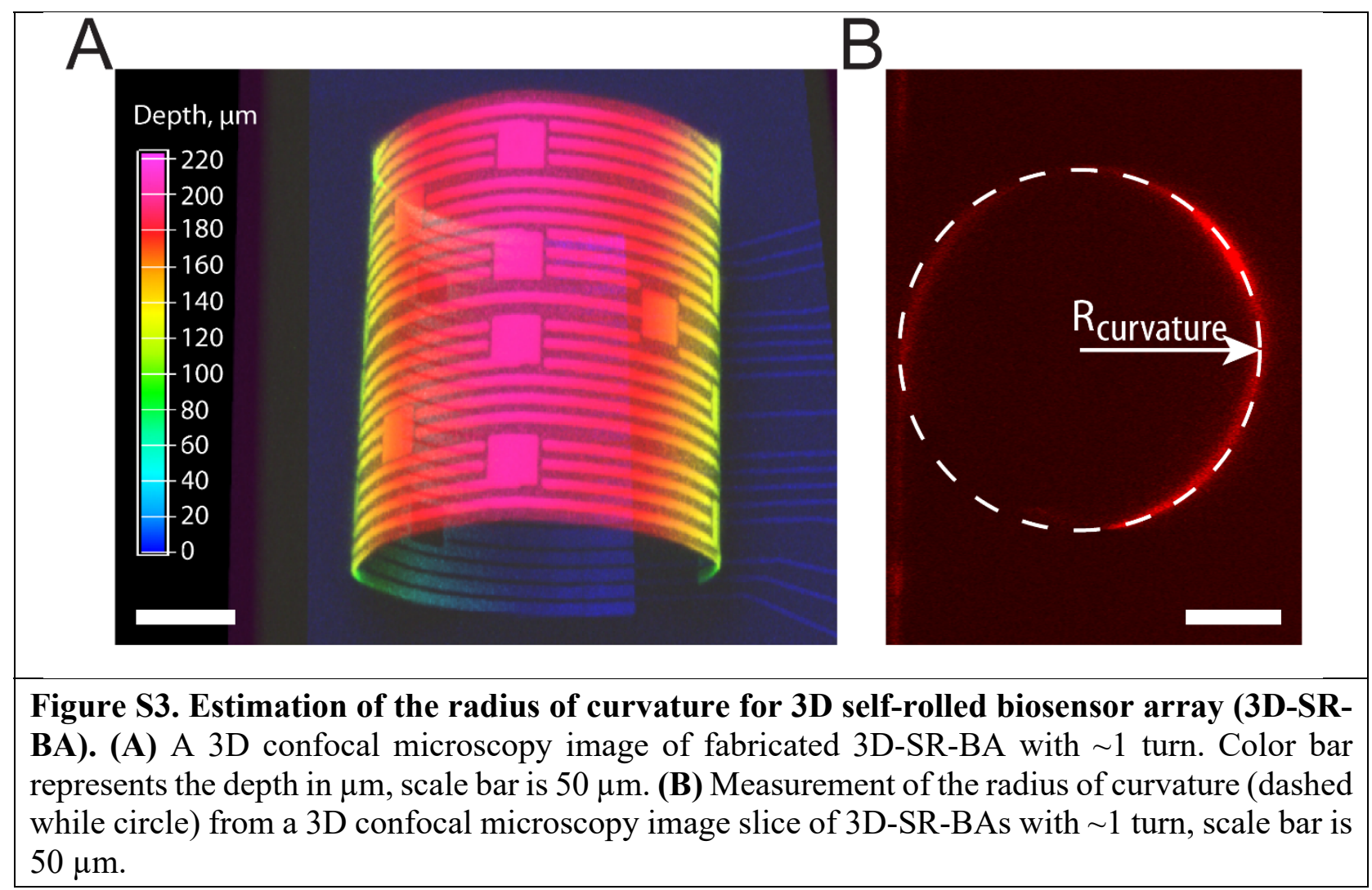




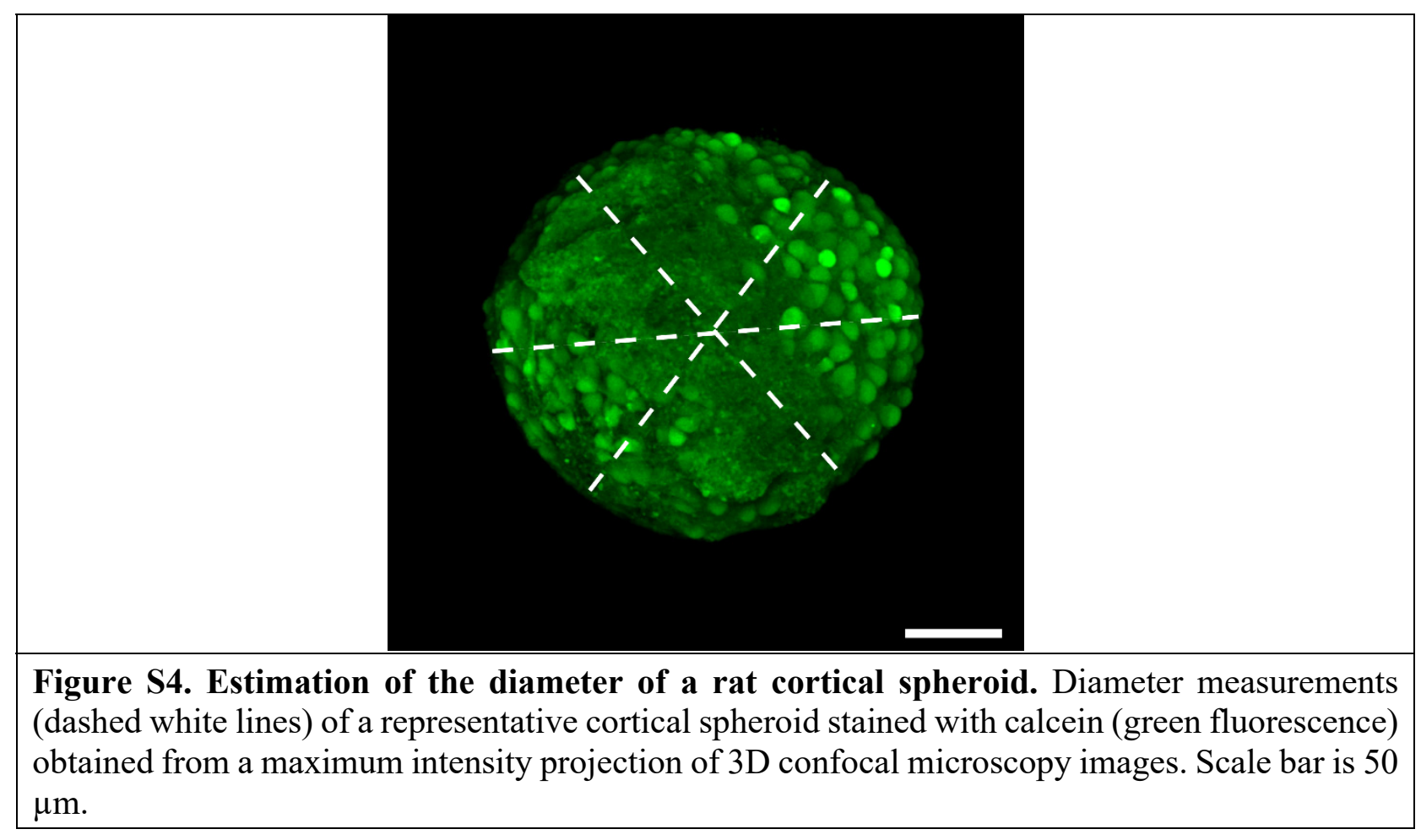


A

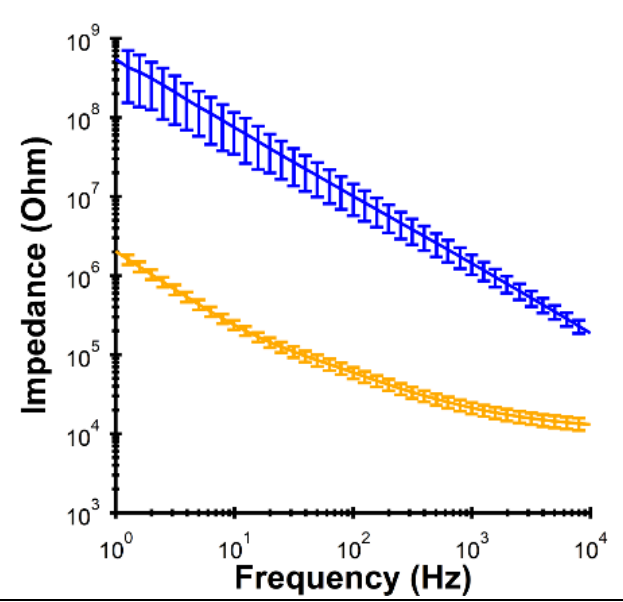

B

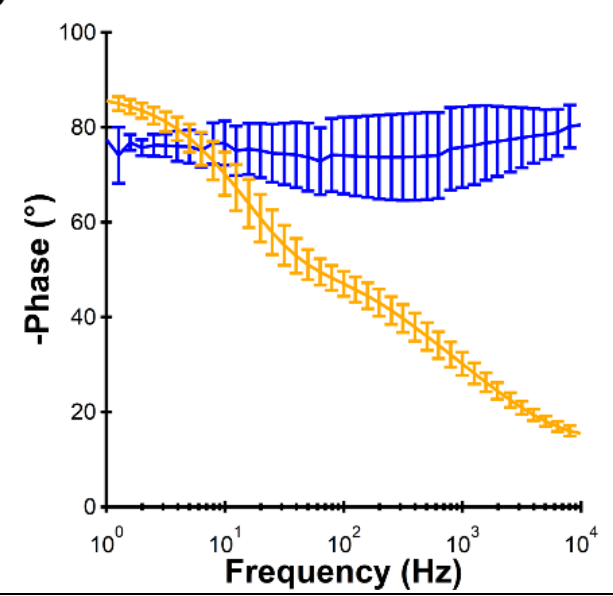

Figure S5. Electrochemical impedance spectroscopy (EIS) characterization of the electrodes before (blue trace) and after (orange trace) PEDOT: PSS electrodeposition. (A) Magnitude of the impedance and (B) Negative phase as a function of frequency. Data is presented as mean \pm $\mathrm{SD}(\mathrm{n}=11$ electrodes$)$. 

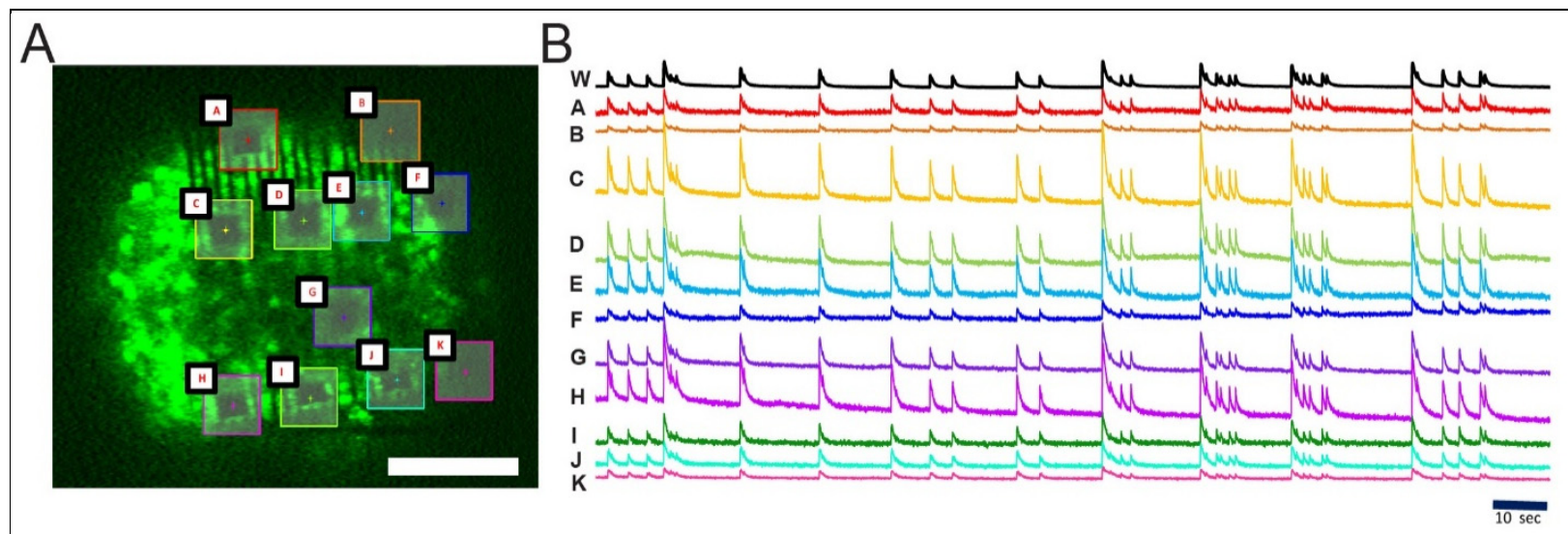

Figure S6. Functional $\mathrm{Ca}^{2+}$ imaging from local regions of interest on cortical spheroid correspond to the whole-tissue optical recording of $\mathrm{Ca}^{2+}$ transients. (A) A $3 \mathrm{D}$ confocal microscopy image of 3D cortical spheroid labeled with $\mathrm{Ca}^{2+}$ indicator dye (Cal-520, green fluorescence) encapsulated by the 3D self-rolled biosensor array (3D-SR-BA). (B) Representative $\mathrm{Ca}^{2+}$ transients from the regions of interest marked by boxes in panel (A). "W" denotes whole-tissue fluorescence. Scale bars are $100 \mu \mathrm{m}$. 


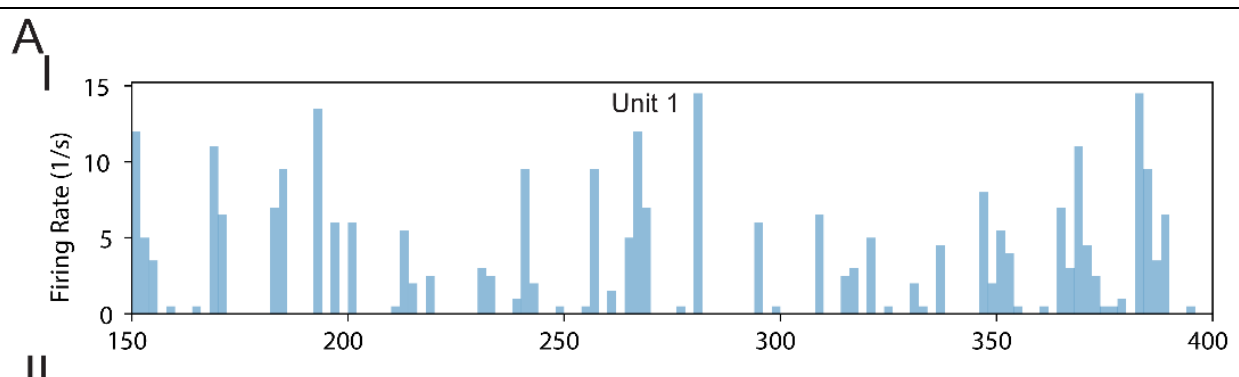

$\|$

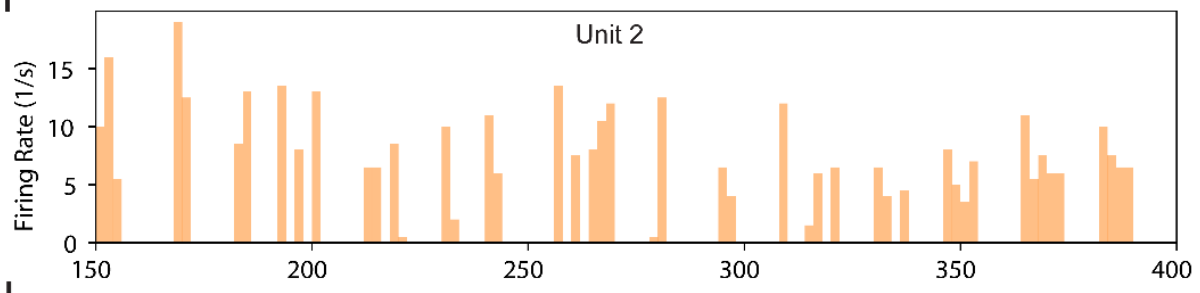

||

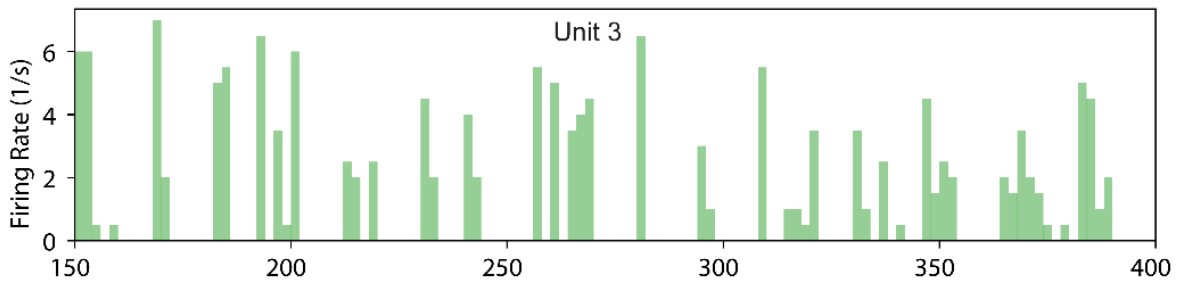

IV
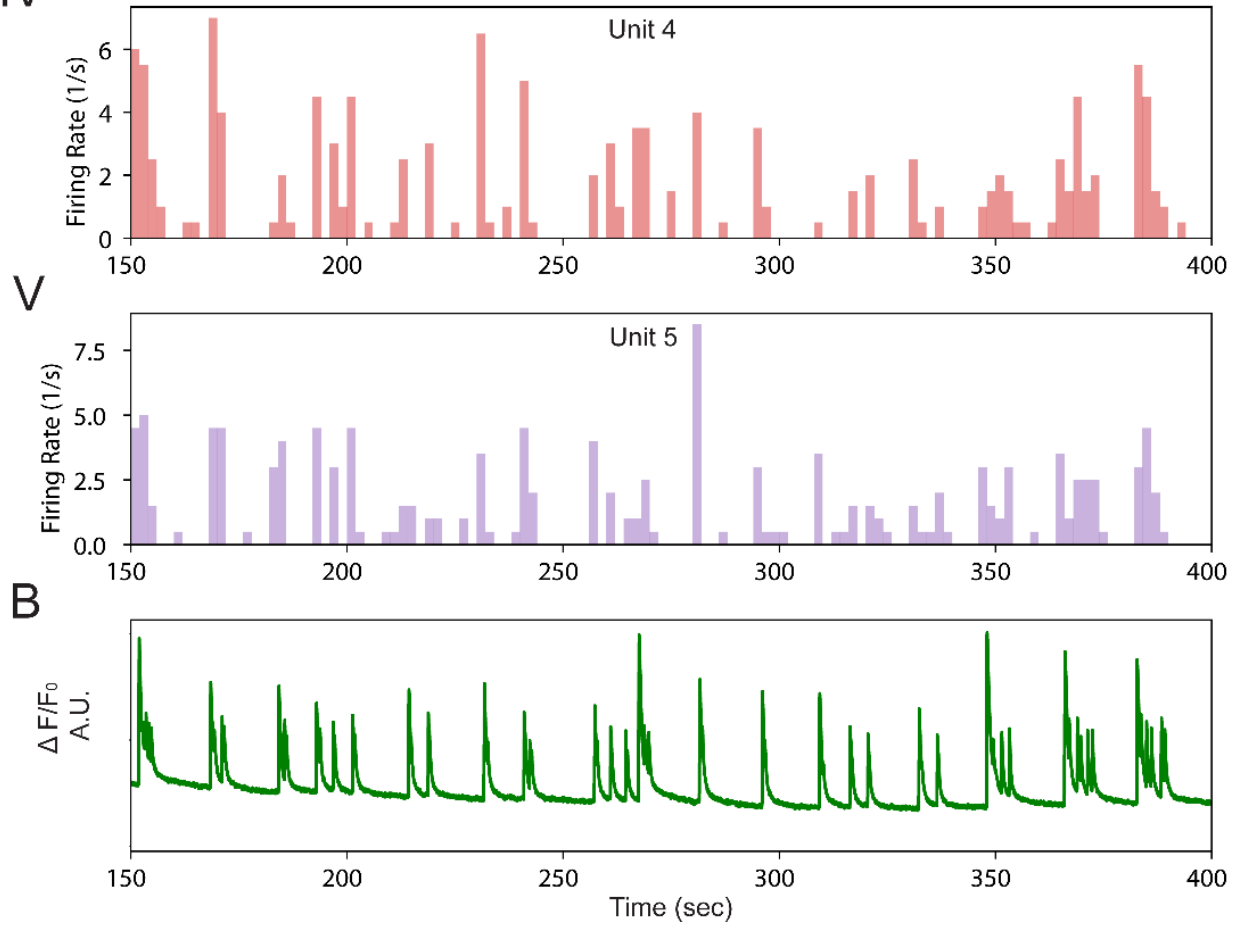

Figure S7. Spontaneous firing of representative units detected after spike sorting of the field potential recording and corresponding whole-spheroid $\mathrm{Ca}^{2+}$ fluorescence. (A) Firing rate of individual units: (I) unit 1, (II) unit 2, (III) unit 3, (IV) unit 4, and (V) unit 5. (B) Optically recorded $\mathrm{Ca}^{2+}$ fluorescence intensity from the same time range. 


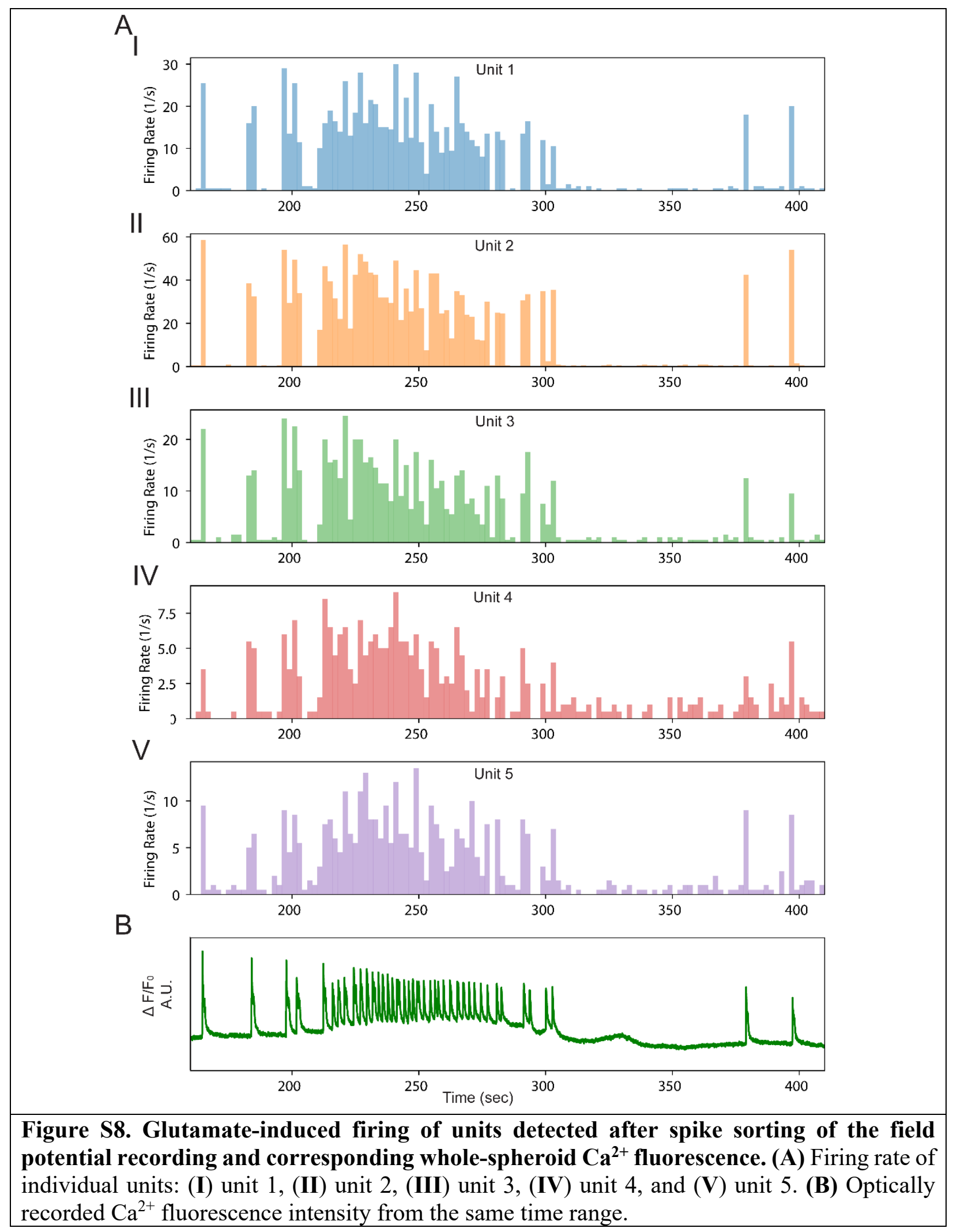




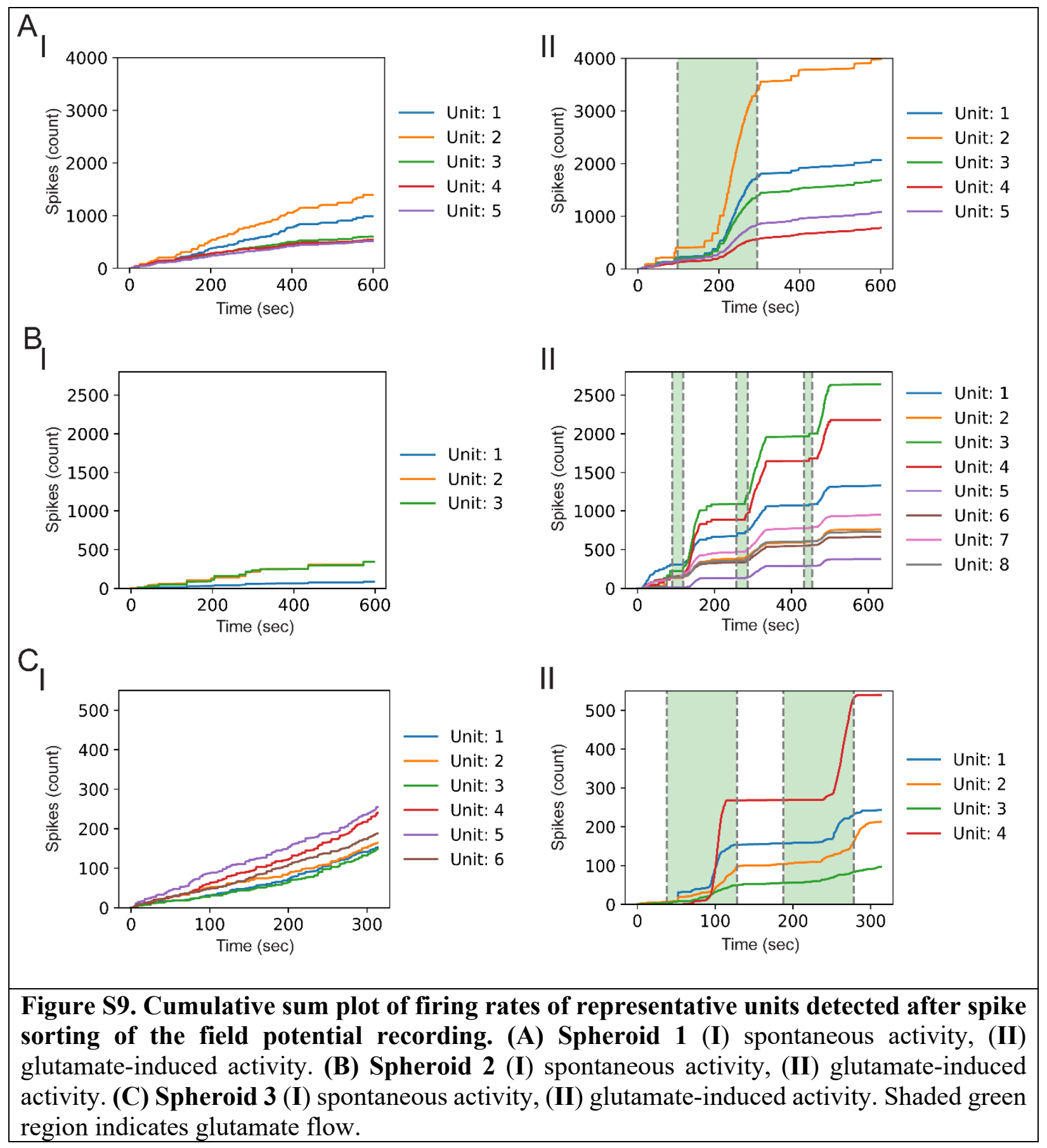




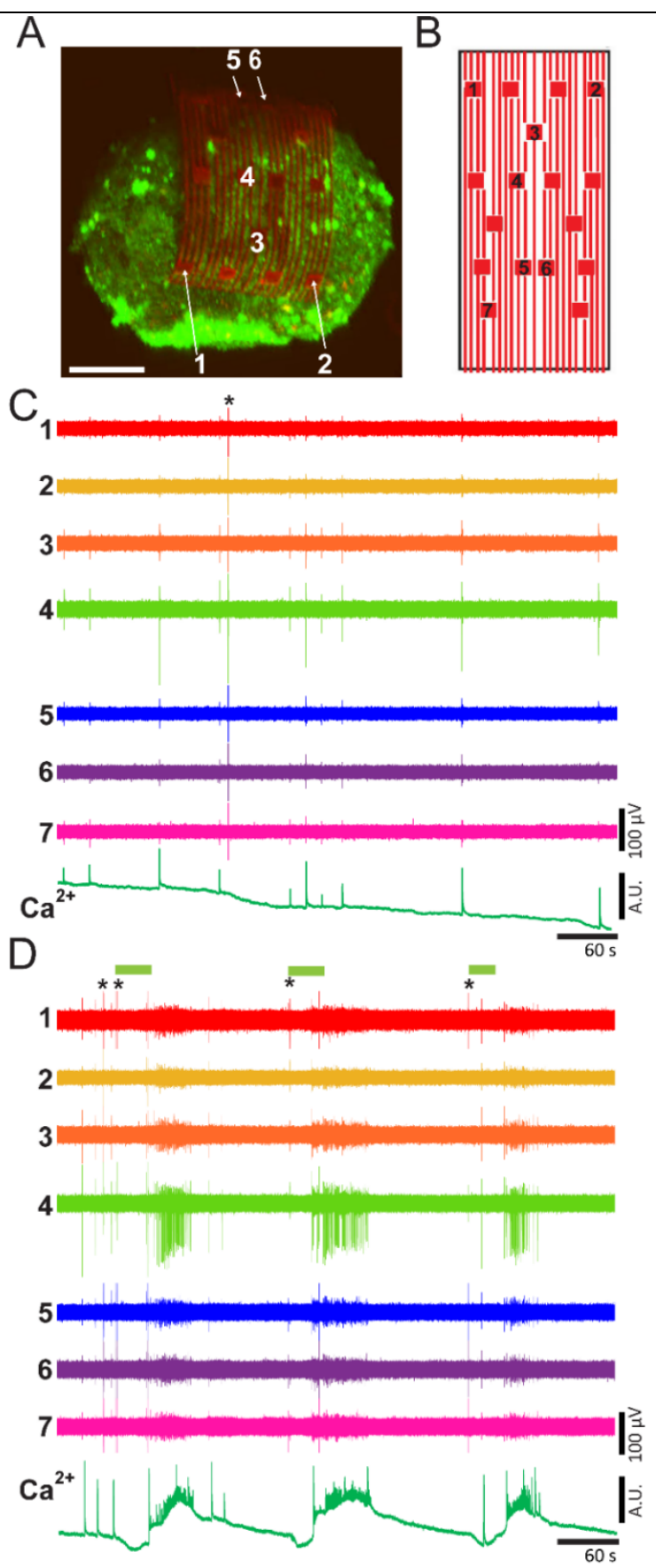

Figure S10. Electrical and optical recordings in 3D of cortical spheroids (second spheroid) using 3D-self-rolled biosensor array (3D-SR-BA). (A) A 3D confocal microscopy image of $3 \mathrm{D}$ cortical spheroid labeled with $\mathrm{Ca}^{2+}$ indicator dye (Cal-520, green fluorescence) encapsulated by the 3D-SR-BA. Scale bar is $100 \mu \mathrm{m}$. (B) A 2D map of the microelectrodes labeled in panel (A) recording electrical activity of cortical neurons. (C) Voltage traces of spontaneously firing cortical neurons recorded from the channels labeled in panel (A) and (B) with simultaneously recorded $\mathrm{Ca}^{2+}$ fluorescence intensity (whole tissue). (D) Voltage traces of cortical neurons firing upon addition of glutamate recorded from the channels labeled in panel (A) and (B) with simultaneously recorded $\mathrm{Ca}^{2+}$ fluorescence intensity (whole spheroid). Green bars indicate glutamate flow. * Denotes electrical artifacts. 


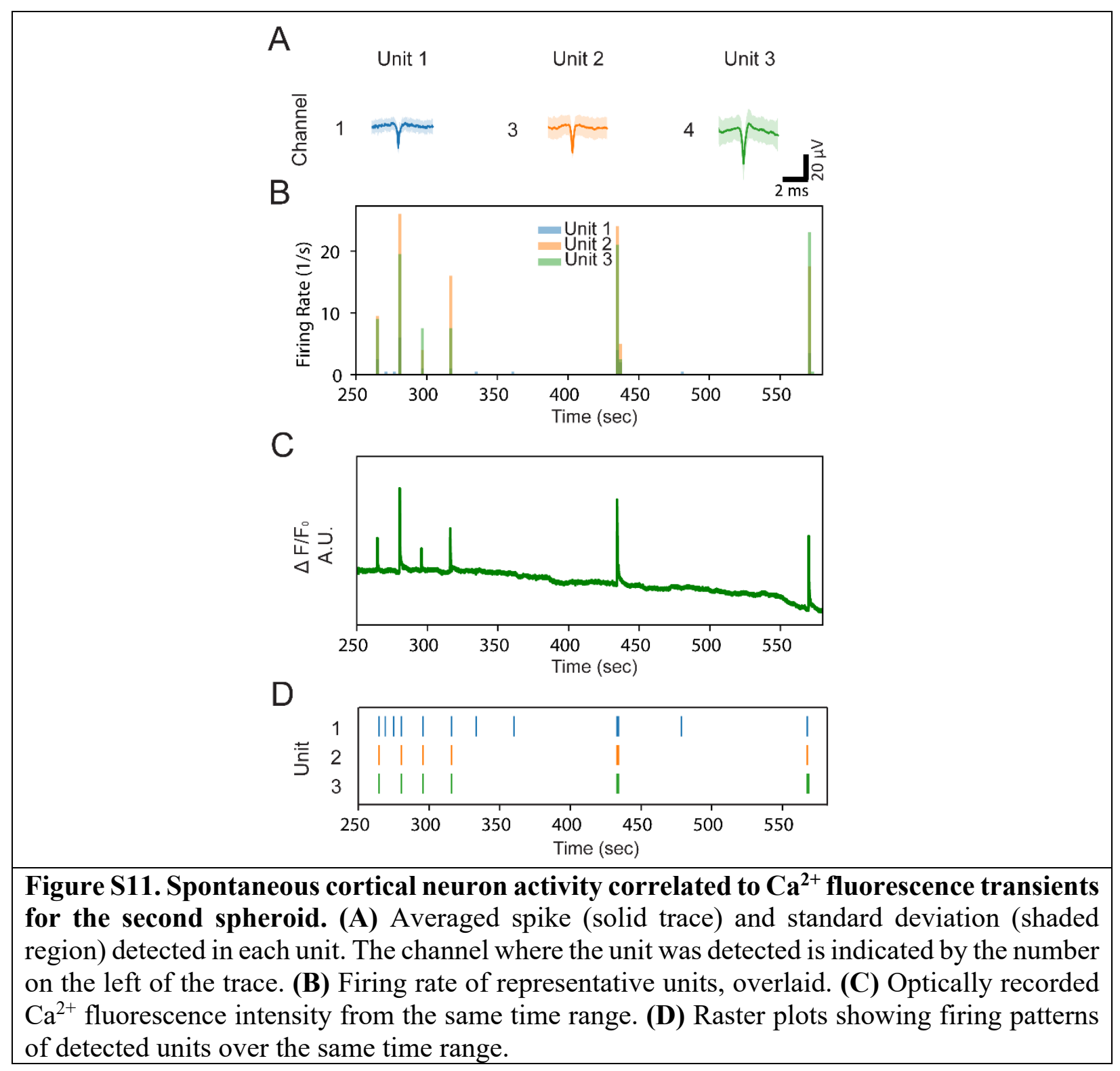




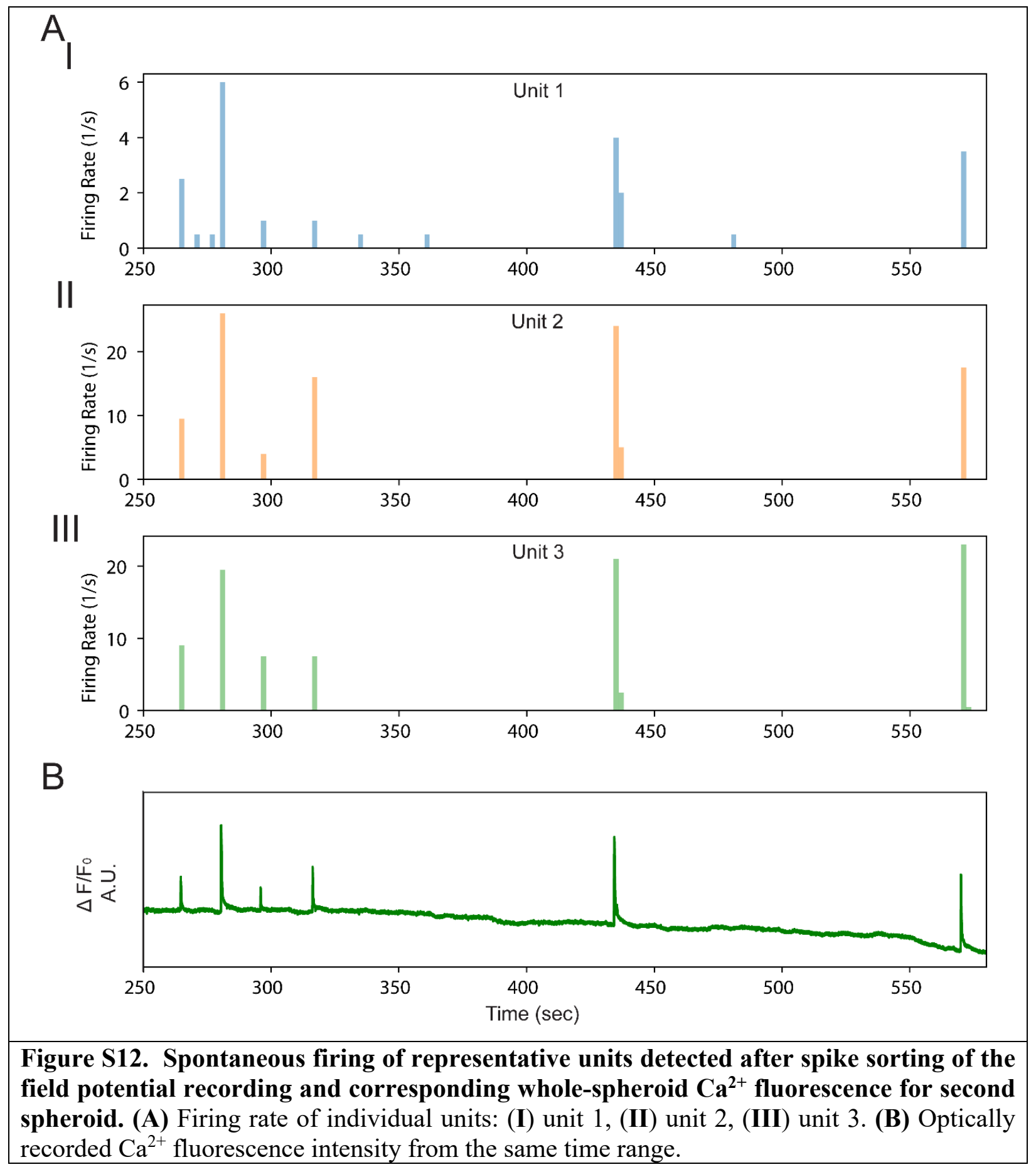




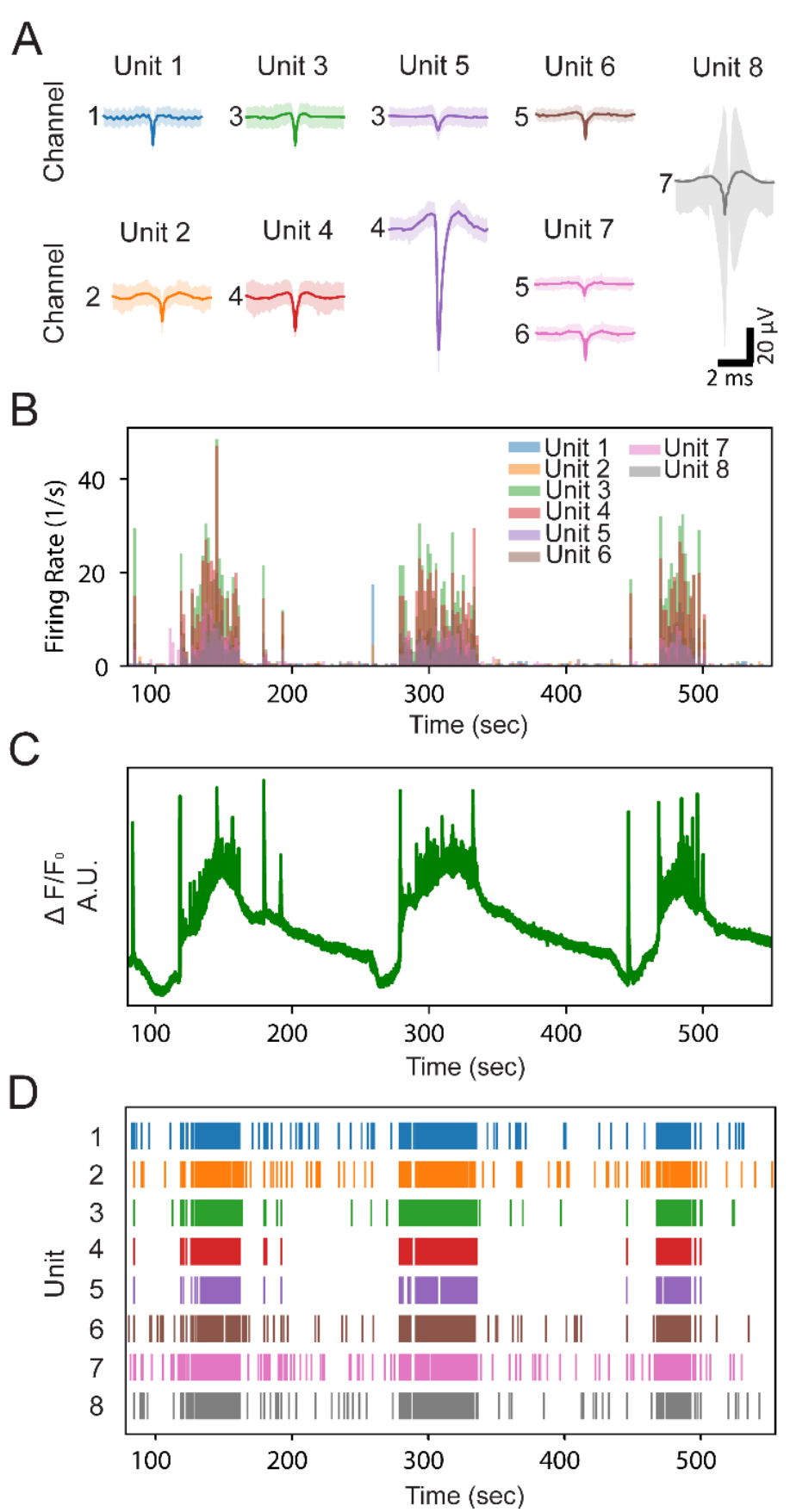

Figure S13. Glutamate-induced cortical neuron activity correlated to $\mathrm{Ca}^{2+}$ fluorescence transients for the second spheroid. (A) Averaged spike (solid trace) and standard deviation (shaded region) detected in each unit. The channel where the unit was detected is indicated by the number on the left of the trace. (B) Firing rate of representative units, overlaid. (C) Optically recorded $\mathrm{Ca}^{2+}$ fluorescence intensity from the same time range. (D) Raster plots showing firing patterns of detected units over the same time range. 


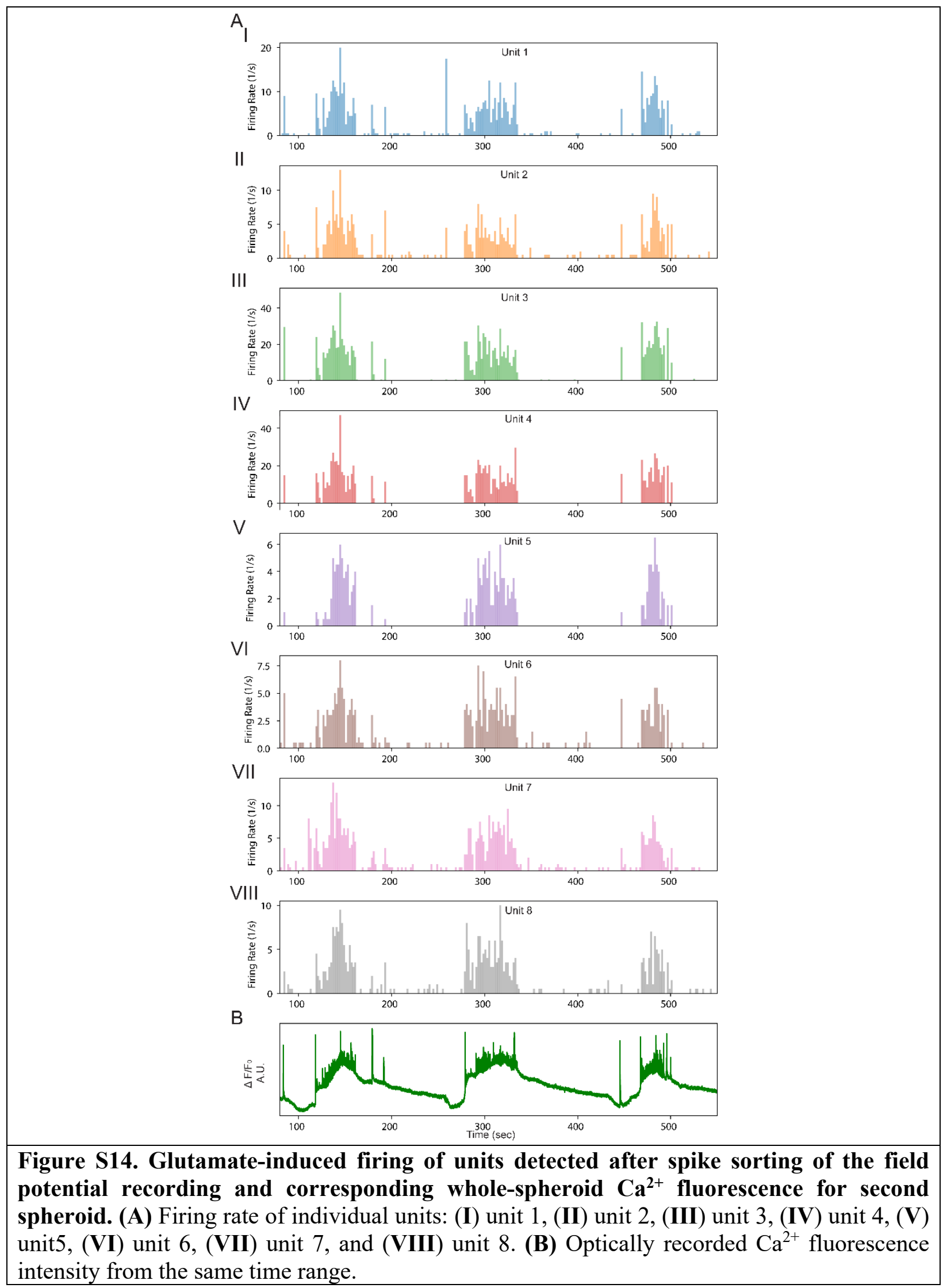




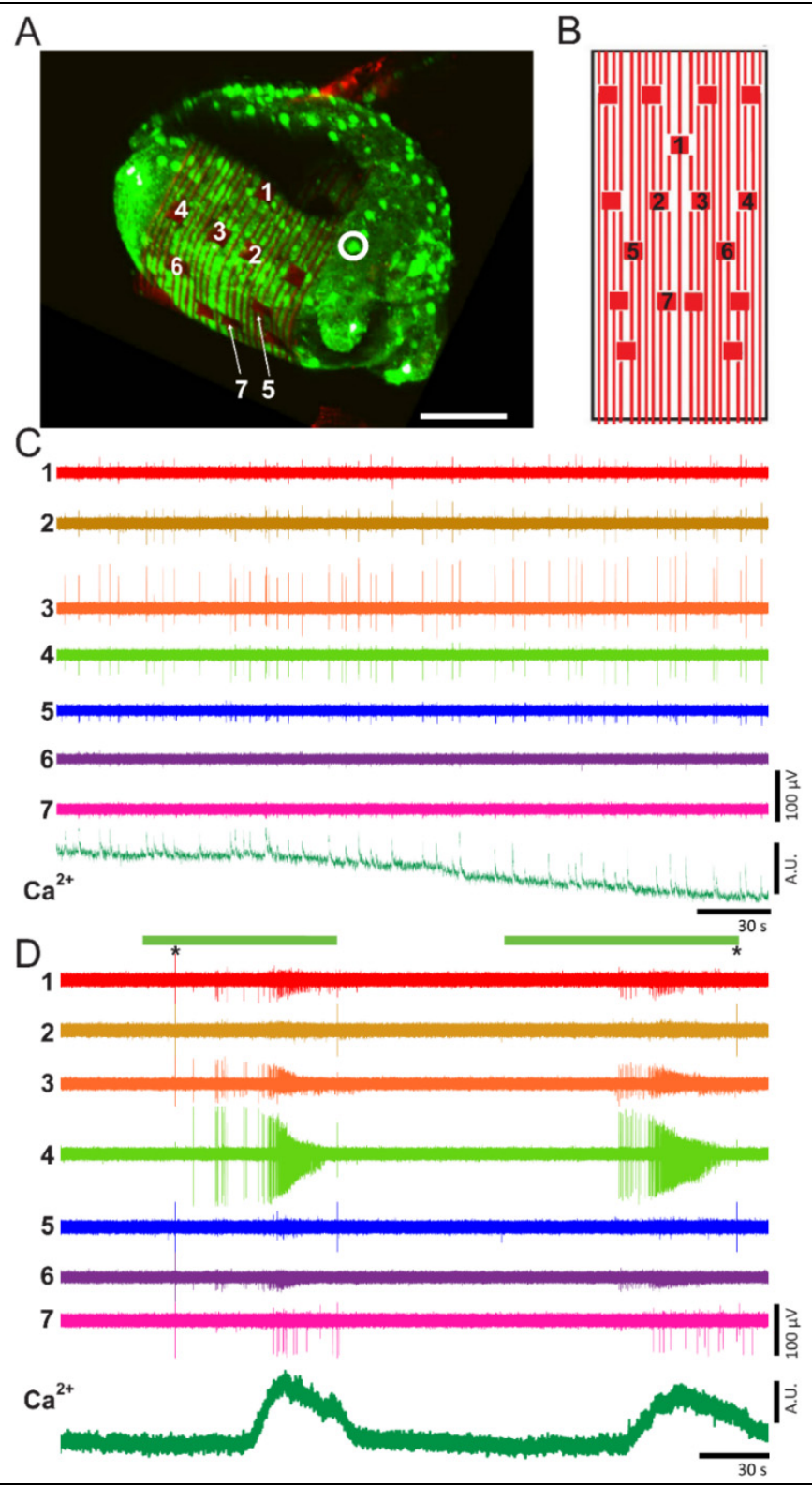

Figure S15. Electrical and optical recordings in 3D of cortical spheroids (third spheroid) using 3D-self-rolled biosensor array (3D-SR-BA). (A) A 3D confocal microscopy image of $3 \mathrm{D}$ cortical spheroid labeled with $\mathrm{Ca}^{2+}$ indicator dye (Cal-520, green fluorescence) encapsulated by the 3D-SR-BA. Scale bar is $100 \mu \mathrm{m}$. (B) A 2D map of the microelectrodes labeled in panel (A) recording electrical activity of cortical neurons. (C) Spontaneously firing neurons recorded via the channels labeled in panel (A) and (B) with simultaneous functional $\mathrm{Ca}^{2+}$ imaging. (D) Voltage traces recorded from cortical neurons firing upon addition of glutamate and simultaneous functional $\mathrm{Ca}^{2+}$ imaging, with fluorescence intensity recorded from a region marked by the white circle. Green bars indicate the time of glutamate flow. * Denotes electrical artifacts. 


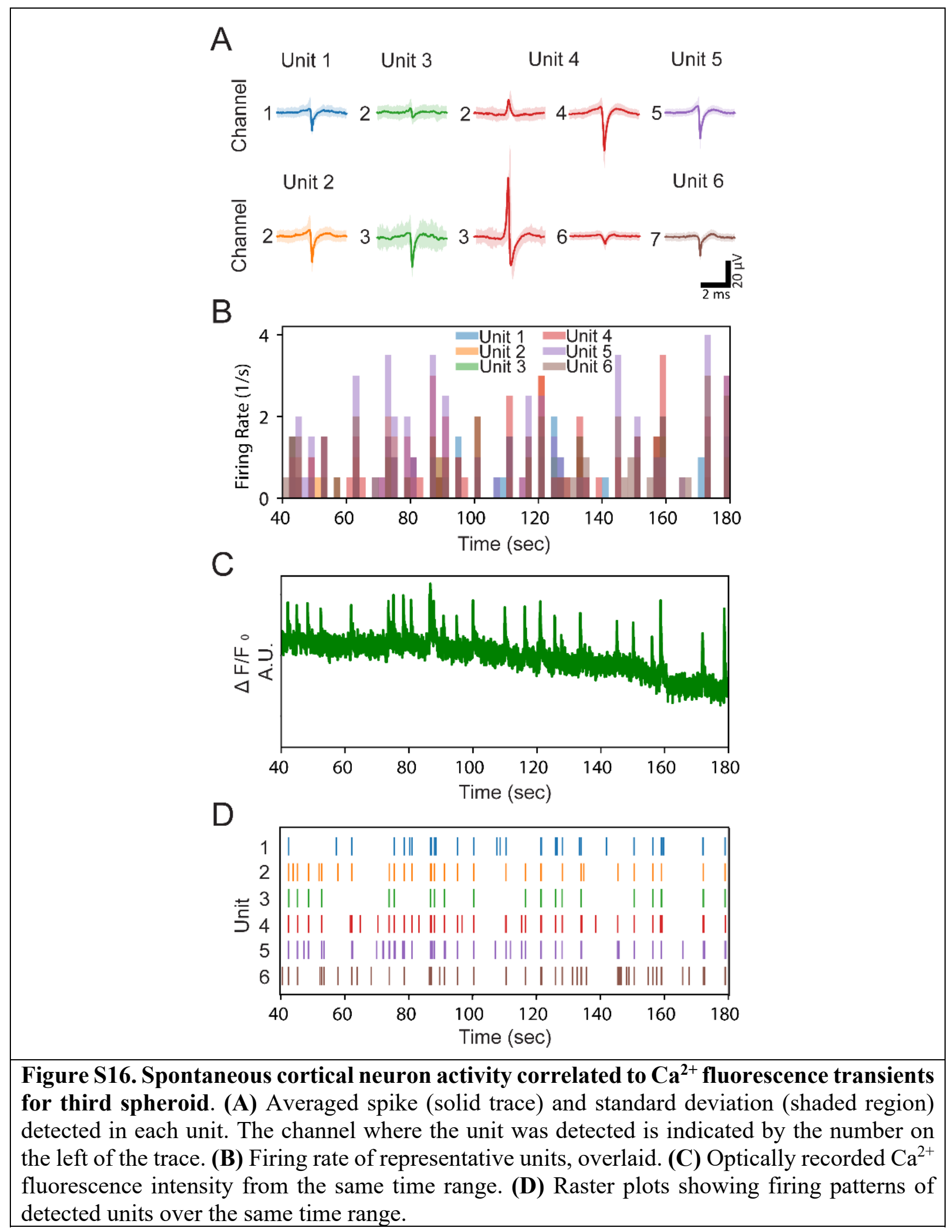



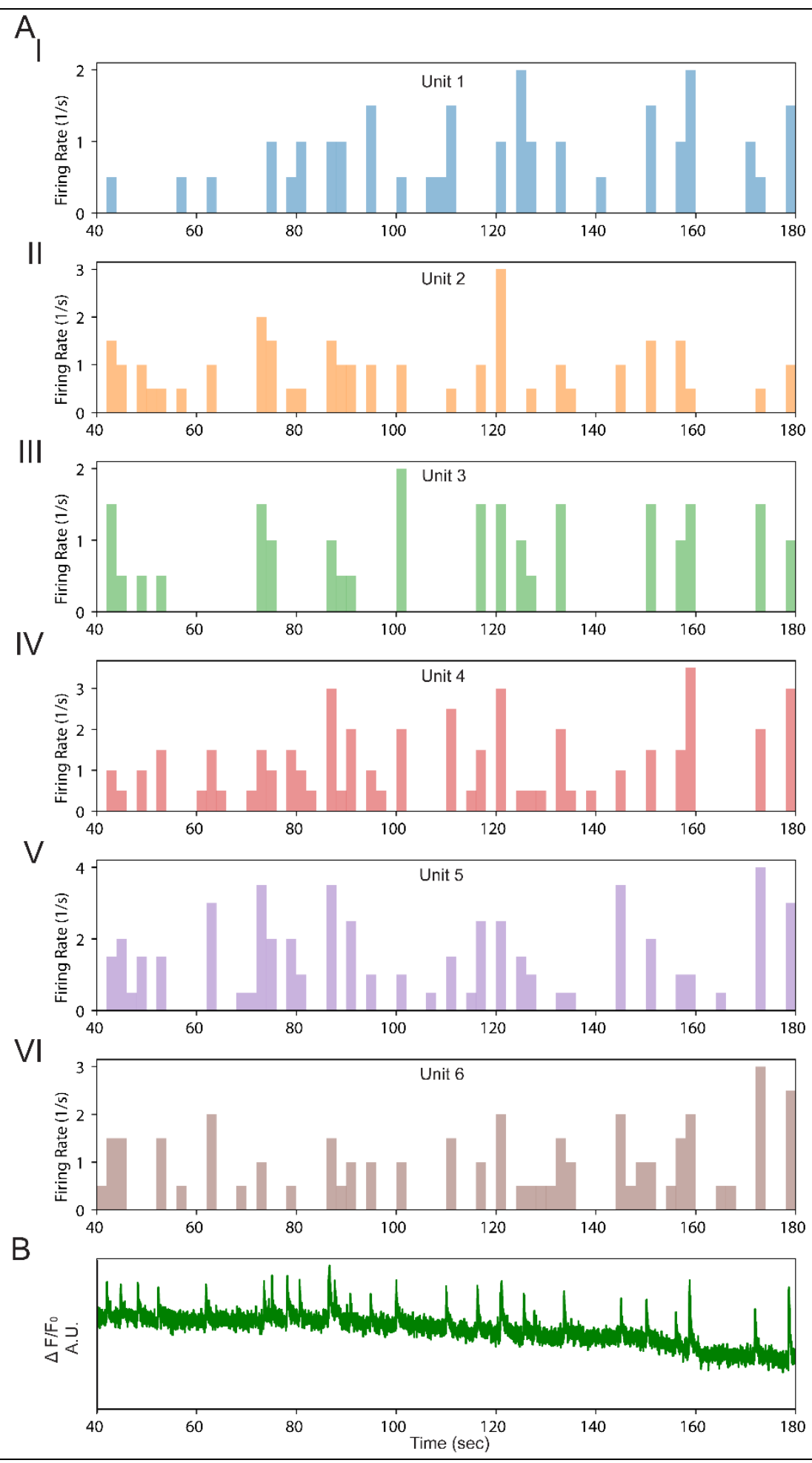

Figure S17. Spontaneous firing of representative units detected after spike sorting of the field potential recording and corresponding whole-spheroid $\mathrm{Ca}^{2+}$ fluorescence for third spheroid. (A) Firing rate of individual units: (I) unit 1, (II) unit 2, (III) unit 3, (IV) unit 4, (V) unit 5, and (VI) unit 6. (B) Optically recorded $\mathrm{Ca}^{2+}$ fluorescence intensity from the same time range. 


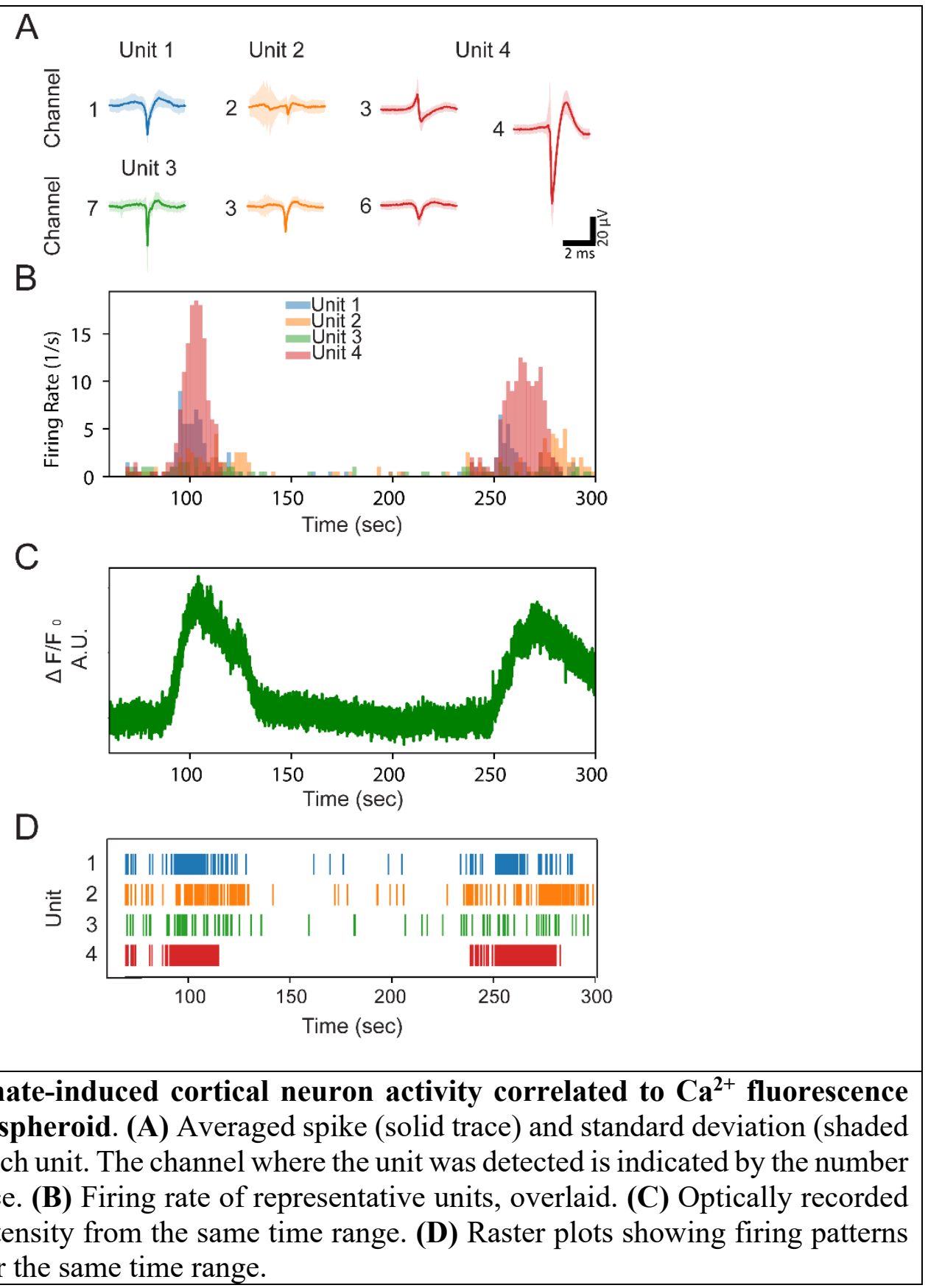

Figure S18. Glutamate-induced cortical neuron activity correlated to $\mathrm{Ca}^{2+}$ fluorescence transients for third spheroid. (A) Averaged spike (solid trace) and standard deviation (shaded region) detected in each unit. The channel where the unit was detected is indicated by the number on the left of the trace. (B) Firing rate of representative units, overlaid. (C) Optically recorded $\mathrm{Ca}^{2+}$ fluorescence intensity from the same time range. (D) Raster plots showing firing patterns of detected units over the same time range. 

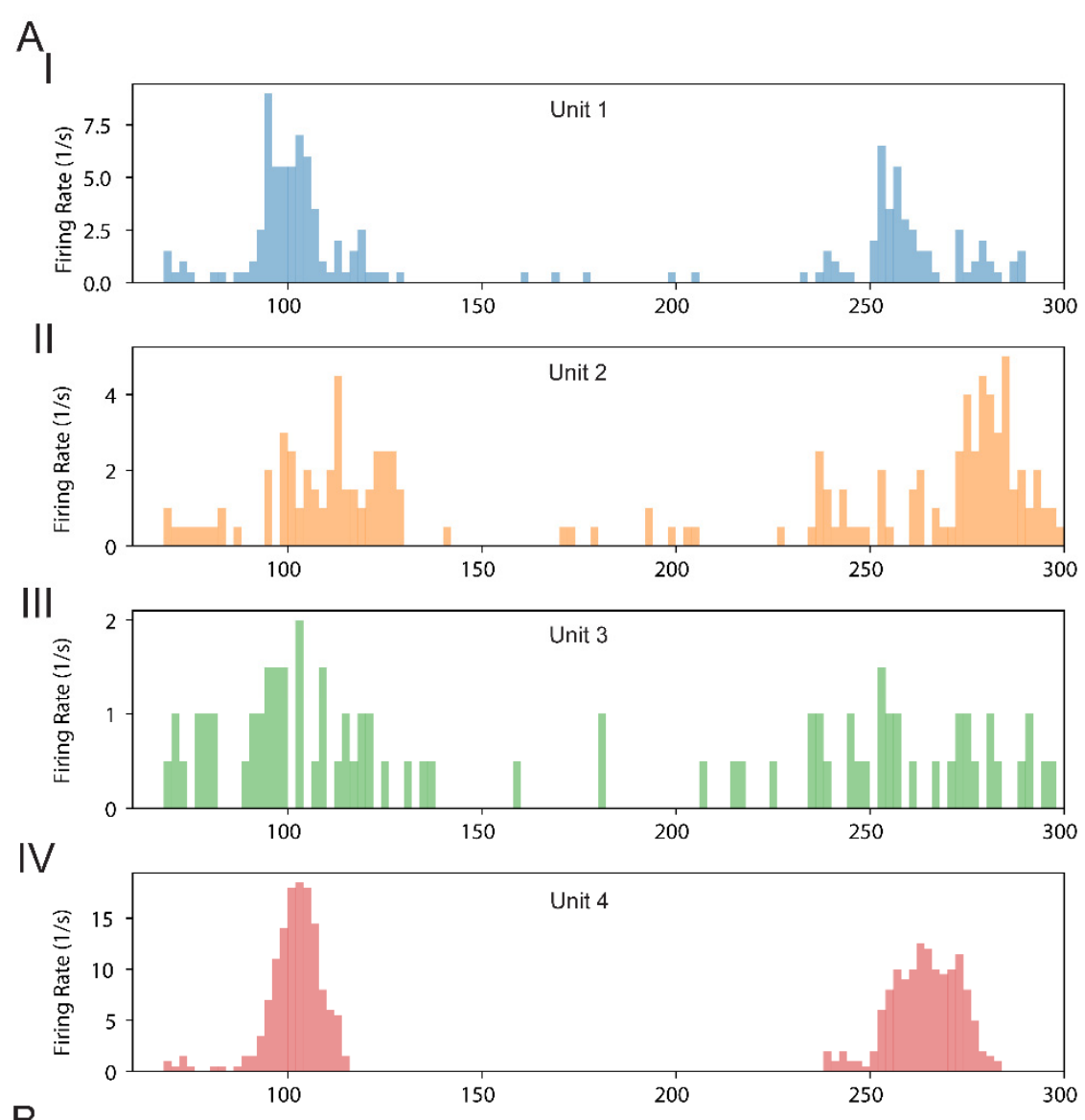

B

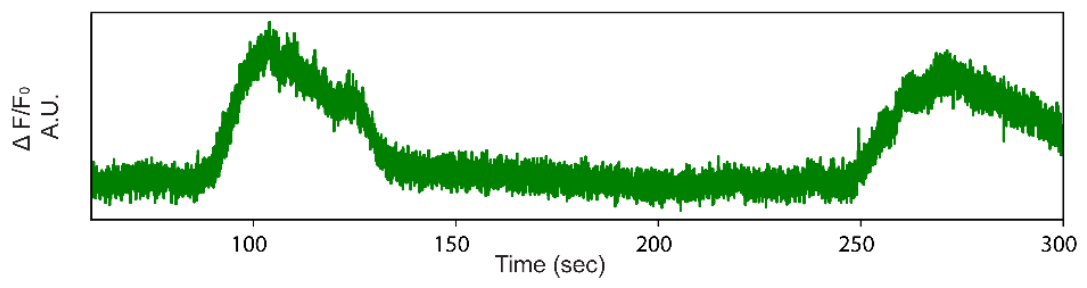

Figure S19. Glutamate-induced firing of representative units detected after spike sorting of the field potential recording and corresponding $\mathrm{ROI} \mathrm{Ca}^{2+}$ fluorescence for third spheroid. (A) Firing rate of individual units: (I) unit 1, (II) unit 2, (III) unit 3, and (IV) unit 4. (B) Optically recorded $\mathrm{Ca}^{2+}$ fluorescence intensity from the same time range. 